\title{
THE DIFFEOMORPHISM TYPE OF SYMPLECTIC FILLINGS
}

\author{
KILIAN BARTH, HANSJÖRG GEIGES, AND KAI ZEHMISCH
}

\begin{abstract}
We show that simply connected contact manifolds that are subcritically Stein fillable have a unique symplectically aspherical filling up to diffeomorphism. Various extensions to manifolds with non-trivial fundamental group are discussed. The proof rests on homological restrictions on symplectic fillings derived from a degree-theoretic analysis of the evaluation map on a suitable moduli space of holomorphic spheres. Applications of this homological result include a proof that compositions of right-handed Dehn twists on Liouville domains are of infinite order in the symplectomorphism group. We also derive uniqueness results for subcritical Stein fillings up to homotopy equivalence and, under some topological assumptions on the contact manifold, up to diffeomorphism or symplectomorphism.
\end{abstract}

\section{Introduction}

The aim of this paper is to study the topology of symplectic fillings $(W, \omega)$ of a given contact manifold $(M, \xi)$. By 'symplectic filling' we always mean a strong filling [14, Definition 5.1.1].

Any filling can of course be modified by performing symplectic blow-ups; this is ruled out if we require the filling $(W, \omega)$ to be symplectically aspherical, that is, $\left.[\omega]\right|_{\pi_{2}(W)}=0$.

It is well known that even under this asphericity assumption symplectic fillings are not, in general, unique up to diffeomorphism. For instance, McDuff [27] observed that the lens space $L(4,1)$ with its standard contact structure coming from the 3 -sphere is Stein fillable both by the disc bundle over the 2-sphere $S^{2}$ with Euler class -4 , and by the complement of the quadric in $\mathbb{C P}^{2}$, cf. [36, Exercises 12.3.4]. Many more examples of lens spaces with non-unique fillings have been found by Lisca [25.

On the other hand, there are also contact manifolds whose symplectic fillings are determined up to diffeomorphism, or even symplectomorphism. The first result about the diffeomorphism type of fillings (in all dimensions) is due to EliashbergFloer-McDuff, see [28, Theorem 1.5]. Here $\xi_{\text {st }}$ denotes the standard contact structure on the odd-dimensional standard sphere coming from the obvious filling by the standard symplectic ball.

Theorem 1.1 (Eliashberg-Floer-McDuff). Let $(W, \omega)$ be a symplectically aspherical filling of $\left(S^{2 n-1}, \xi_{\mathrm{st}}\right), n \geq 3$. Then $W$ is diffeomorphic to the ball $D^{2 n}$.

Earlier, it had been proved by Gromov [20, p. 311] and McDuff [27, Theorem 1.7], using positivity of intersection in dimension four, that any symplectically aspherical filling of $\left(S^{3}, \xi_{\mathrm{st}}\right)$ is even symplectomorphic to the standard 4-ball.

2010 Mathematics Subject Classification. 57R17; 32Q65, 53D35, 57R65, 57R80.

H. G. and K. Z. are partially supported by DFG grants GE 1245/2-1 and ZE 992/1-1, respectively. 
In this paper, we study the topology of symplectically aspherical fillings of contact manifolds that admit a subcritical Stein filling, that is, where the plurisubharmonic function on the Stein filling has handles of index below the middle dimension only. Essentially, what we show (under various topological assumptions) is that the existence of a single subcritical Stein filling fixes the diffeomorphism type of all symplectically aspherical fillings.

By analysing the moduli space of holomorphic spheres in a partial compactification of the filling, we derive a degree-theoretic statement concerning the evaluation map on this moduli space. This approach was pioneered by McDuff in [28], and developed further by two of the present authors in a number of papers, e.g. [16, 17, 18, From the evaluation map on the moduli space we derive a homological vanishing result for fillings, which then leads to the following result. Here, by slight abuse of notation, we write the Stein filling as a pair $\left(W_{0}, \omega_{0}\right)$ consisting of a manifold and a symplectic form, since we are primarily interested in the symplectic properties of fillings.

Theorem 1.2. Let $(M, \xi)$ be a $(2 n-1)$-dimensional closed, connected contact manifold, $n \geq 2$, admitting a subcritical Stein filling $\left(W_{0}, \omega_{0}\right)$ with the homotopy type of a $C W$ complex of dimension $\ell_{0} \leq n-1$. Let $(W, \omega)$ be any symplectically aspherical filling of $(M, \xi)$. Then the following holds:

(a) The integral homology groups of $W$ are

$$
H_{k}(W) \cong \begin{cases}H_{k}(M) & \text { for } k=0, \ldots, \ell_{0} \\ 0 & \text { otherwise }\end{cases}
$$

where the isomorphism between the relevant homology groups of $M$ and $W$ is induced by the inclusion $M \subset W$. In particular, the homology groups of $W$ coincide with those of $W_{0}$.

(b) The inclusion $M \subset W$ is $\pi_{1}$-surjective. If the fundamental group $\pi_{1}(M)$ is abelian, then the inclusion $M \subset W$ is also $\pi_{1}$-injective.

This theorem will be proved in Section 2. Various direct applications are discussed in Section 3

Remark 1.3. In the proof of Theorem 1.2, we appeal to a result of Cieliebak, which relies on the assumption that the filling is subcritical as a Stein manifold, i.e. there are no Stein handles of critical index. It is probably not sufficient, in general, merely to assume that the Stein filling is of subcritical homotopical dimension (that is, where the critical handles cancel topologically). Stein manifolds of this kind exist by the work of Seidel-Smith [41] and McLean [31]: in all even dimensions $2 n \geq 8$ there are infinitely many distinct finite type Stein manifolds diffeomorphic to $\mathbb{R}^{2 n}$; they all have Stein handle decompositions involving critical handles.

Our most significant application of Theorem 1.2 is the following vast extension of results of Seidel on generalised Dehn twists. This is discussed in Section 4, where all the relevant concepts will be introduced. For other work in this direction see Remark 4.6.

Theorem 1.4. A (non-empty) composition of right-handed Dehn twists on a Liouville manifold of dimension at least four is never isotopic to the identity within the group of compactly supported symplectomorphisms. 
Theorem 1.2 is also one of the essential steps towards the main result of this paper, proved in Section 5, about the topological classification of symplectic fillings.

Theorem 1.5. Let $(M, \xi)$ be as in Theorem 1.2, $n \geq 3$. If $M$ is simply connected, then all symplectically aspherical fillings of $(M, \xi)$ are diffeomorphic.

An extension of this result to certain finite fundamental groups is given in Theorem 5.3

In Section 6 we generalise the argument used to prove Theorem 1.2 to the setting of coverings. This is used in Section 7 to derive results on the homotopy and diffeomorphism type of fillings when the maximal index of a handle decomposition of $W$ is known. One result that is easy to state is the following.

Theorem 1.6. All subcritical Stein fillings of a closed, connected contact manifold are homotopy equivalent.

Of course the statement may be empty if the given contact manifold does not admit any subcritical Stein fillings.

In Section 8 we consider fillings of simple manifolds $M$. Recall that a topological space is called simple if its fundamental group acts trivially on all its homotopy groups. Examples of simple manifolds are Lie groups or, more generally, any manifold that is an $H$-space [26, Corollary $\left.8^{\text {bis }} .3\right]$. The main result of Section 8 is the following.

Theorem 1.7. Let $(M, \xi)$ be as in Theorem 1.2, $n \geq 3$. If $M$ is a simple space whose fundamental group has vanishing Whitehead group, then all symplectically aspherical fillings of $M$ are diffeomorphic.

In Section 9 we apply our theory to the sphere bundle of stabilised cotangent bundles. Finally, in Section 10 we show that if $(M, \xi)$ admits a 2-subcritical Stein filling, then all flexible Stein fillings (e.g. subcritical ones) are symplectomorphic.

For other results on the topology of Stein fillings see [9, Chapter 16] and [36, Chapter 12] and the references therein.

\section{Proof of Theorem 1.2}

2.1. A completion of the filling. According to a theorem of Cieliebak [8, , 9 , Section 14.4], every subcritical Stein manifold is deformation equivalent (hence symplectomorphic) to a split one. Thus, if $\left(W_{0}, \omega_{0}\right)$ is the given subcritical Stein filling of $(M, \xi)$, we may assume - perhaps after scaling $\omega_{0}$ by a small positive constant - that there is a $(2 n-2)$-dimensional Stein manifold $\left(V, J_{V}\right)$ with plurisubharmonic function $\psi_{V}\left(\right.$ with $\left.\min \psi_{V}=0\right)$ and symplectic form $\omega_{V}=-\mathrm{d}\left(\mathrm{d} \psi_{V} \circ J_{V}\right)$, such that $W_{0}$ is a sublevel set of the plurisubharmonic potential

$$
\psi(v, z):=\psi_{V}(v)+\frac{1}{4} \log \left(1+|z|^{2}\right)
$$

on $\left(V \times \mathbb{C}, J_{V} \oplus \mathrm{i}\right)$, and such that, with $z=r \mathrm{e}^{\mathrm{i} \theta}$, the corresponding symplectic form

$$
\omega:=\omega_{V}+\frac{r \mathrm{~d} r \wedge \mathrm{d} \theta}{\left(1+r^{2}\right)^{2}}
$$

on $V \times \mathbb{C}$ coincides with $\omega_{0}$ on $W_{0}$ under the inclusion $W_{0} \subset V \times \mathbb{C}$. 
Now, given a symplectically aspherical filling $(W, \omega)$ of $(M, \xi)$, we define the symplectic manifold

$$
(Z, \Omega):=(W, \omega) \cup_{(M, \xi)}\left((V \times \mathbb{C}) \backslash \operatorname{Int}\left(W_{0}\right), \omega_{V}+\frac{r \mathrm{~d} r \wedge \mathrm{d} \theta}{\left(1+r^{2}\right)^{2}}\right) .
$$

Our choice of plurisubharmonic potential on the $\mathbb{C}$-factor is explained by the fact that we can now build a new symplectic manifold $(\hat{Z}, \hat{\Omega})$ from $(Z, \Omega)$ by compactifying $\mathbb{C}$ to a complex projective line $\mathbb{C P}^{1}=\mathbb{C} \cup\{\infty\}$ with its standard Fubini-Study symplectic form of total area $\pi$, see Figure 1.

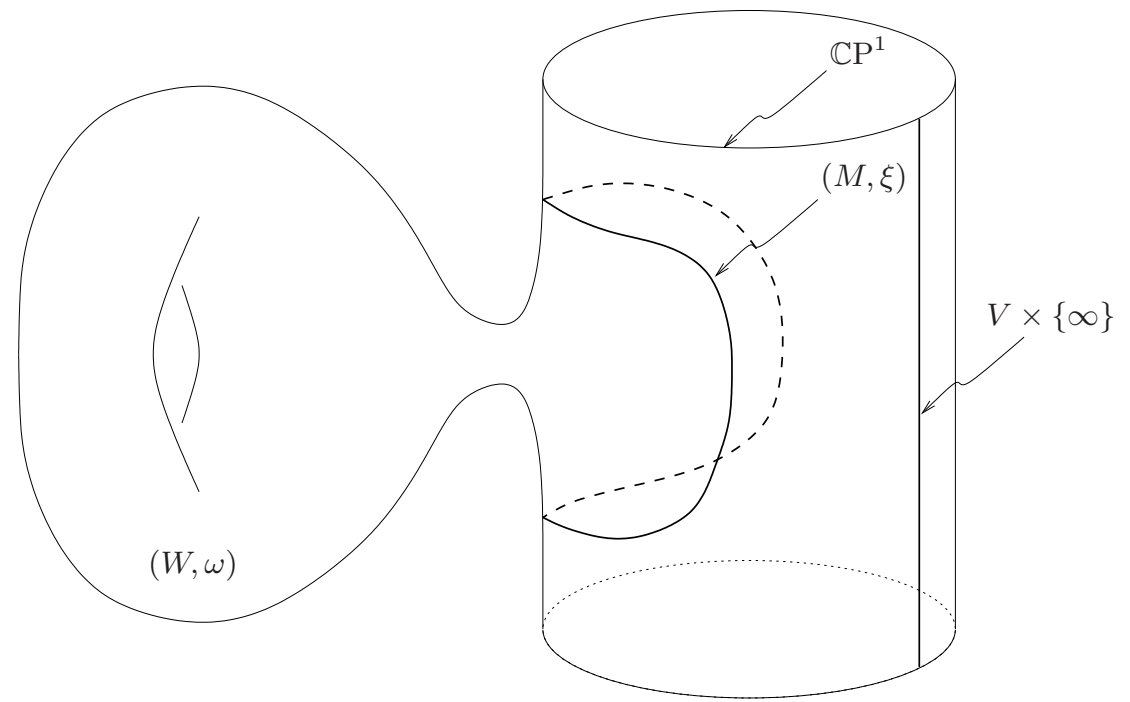

Figure 1. The symplectic manifold $\hat{Z}$.

With $\omega_{0}$ scaled sufficiently small, we may assume that $M=\partial W_{0}$ is a level set of $\psi$ below $(\log 2) / 4$, so that the hypersurfaces $V \times\{ \pm 1\}$ may also be regarded as subsets of $\hat{Z}$. Moreover, without loss of generality we may assume that $\psi_{V}$ has no critical points above the level of $M$. This ensures that the homotopical dimension of $V$ equals that of $W_{0}$, i.e. $\ell_{0}$, and $W$ is a strong deformation retract of $Z$.

We equip the symplectic manifold $(\hat{Z}, \hat{\Omega})$ with a compatible almost complex structure $J$, generic in the sense of [30] on $\operatorname{Int}\left(W_{0}\right)$, and equal to $J_{V} \oplus \mathrm{i}$ on $\hat{Z} \backslash \operatorname{Int}(W)$.

2.2. Holomorphic spheres. For $v \in V$ with $\psi_{V}(v)>(\log 2) / 4$ we have the obvious holomorphic spheres $\{v\} \times \mathbb{C P}^{1} \subset(\hat{Z}, J)$, which foliate the corresponding part of $\hat{Z}$.

Lemma 2.1. Let $u: \mathbb{C P}^{1} \rightarrow \hat{Z}$ be a non-constant J-holomorphic sphere.

(i) If $u\left(\mathbb{C P}^{1}\right)$ is contained in $\hat{Z} \backslash \operatorname{Int}(W)$, then $u$ is a holomorphic branched covering $\mathbb{C P}^{1} \rightarrow\{v\} \times \mathbb{C P}^{1}$ for some $v \in V$.

(ii) If $u\left(\mathbb{C P}^{1}\right)$ intersects $\hat{Z} \backslash \operatorname{Int}(W)$, then it also intersects the hypersurface $H:=V \times\{\infty\}$.

(iii) If $u\left(\mathbb{C P}^{1}\right)$ intersects $\left\{\psi_{V}>(\log 2) / 4\right\} \times \mathbb{C P}^{1}$, then $u$ is as in (i). 
Proof. (i) This follows from the maximum principle for $J_{V}$-holomorphic curves in $V$, applied to the $V$-component of $u$.

(ii) The plurisubharmonic function $\psi$ is defined on $\hat{Z} \backslash(\operatorname{Int}(W) \cup H)$ and a collar neighbourhood of $M$ in $W$, and the symplectic form $\omega$ is exact in this region. Thus, if $u\left(\mathbb{C P}^{1}\right)$ intersects $\hat{Z} \backslash \operatorname{Int}(W)$, but not the hypersurface $H$, the maximum principle constrains $u\left(\mathbb{C P}^{1}\right)$ to lie in a level set of $\psi$, and hence inside the region where $\omega$ is exact. This forces $u$ to be constant.

(iii) Apply the maximum principle to the $V$-component of $u$ on the preimage of $\left\{\psi_{V}>(\log 2) / 4\right\} \times \mathbb{C P}^{1}$.

Let $\mathcal{M}$ be the moduli space of holomorphic spheres $u: \mathbb{C P}^{1} \rightarrow(\hat{Z}, J)$ with the properties

(M1) $[u]=\left[\{v\} \times \mathbb{C P}^{1}\right]$ in the homology group $H_{2}(\hat{Z})$, where $v \in V$ can be any point with $\psi_{V}(v)>(\log 2) / 4$

(M2) $u(z) \in V \times\{z\}$ for $z \in\{ \pm 1, \infty\}$.

Observe that a holomorphic sphere $u \in \mathcal{M}$ satisfying one of the assumptions (i) or (iii) in Lemma 2.1 is simply an inclusion map $\mathbb{C P}^{1} \rightarrow\{v\} \times \mathbb{C P}^{1}$.

Proposition 2.2. The moduli space $\mathcal{M}$ is an oriented manifold of dimension $2 n-2$.

Proof. This is proved exactly as [16. Proposition 6.1]. Notice that our moduli space $\mathcal{M}$ corresponds to $\mathcal{M}_{-1,1, \infty}$ in [16, p. 277].

In the following proposition, the degree of a proper map between non-compact oriented manifolds is understood in the sense of [22, Exercise 5.1.10]. We think of $\mathbb{C P}^{1}$ as $\mathbb{C} \cup\{\infty\}$.

Proposition 2.3. The evaluation map

$$
\begin{array}{clc}
\mathcal{M} \times \mathbb{C P}^{1} & \longrightarrow & \hat{Z} \\
(u, z) & \longmapsto & \longrightarrow(z)
\end{array}
$$

is proper and of degree 1 . It restricts to a proper degree 1 map

$$
\text { ev }: \mathcal{M} \times \mathbb{C} \longrightarrow Z \text {. }
$$

Proof. Let $\left(u^{\alpha}\right)_{1 \leq \alpha \leq N}$ be a stable map in the sense of [30] that arises as the Gromovlimit of a sequence $\left(u_{\nu}\right)$ of spheres in $\mathcal{M}$. We need to show that $N=1$, so that $u_{\nu} \rightarrow u^{1}$ as a $C^{\infty}$-limit. The claim about the first evaluation map then follows from Lemma 2.1 and the observation following the definition of $\mathcal{M}$, which say that the non-compact ends of $\hat{Z}$ are foliated by holomorphic spheres $\{v\} \times \mathbb{C P}^{1}$, and no other spheres intersect these ends.

By positivity of intersections [11, Proposition 7.1] and (M1) we may assume that $u^{1} \bullet H=1$ and $u^{j} \bullet H=0$ for $j=2, \ldots, N$. Then Lemma 2.1 (ii) tells us that the $u^{j}$ are contained entirely in $\operatorname{Int}(W)$ for $j=2, \ldots, N$, but $W$ does not contain any non-constant holomorphic spheres.

From positivity of intersection and (M2) we conclude that $u^{-1}(H)=\{\infty\}$ for $u \in \mathcal{M}$, so the evaluation map restricts to $\mathbb{C} \subset \mathbb{C P}^{1}$ as claimed.

2.3. A homology epimorphism. From Proposition 2.3 we now deduce crucial homological information. 
Proposition 2.4. The induced homomorphism

$$
\mathrm{ev}_{*}: H_{k}(\mathcal{M} \times \mathbb{C}) \longrightarrow H_{k}(Z)
$$

is surjective in all degrees $k$.

Proof. Write $D_{R}^{2} \subset \mathbb{C}$ for the closed 2-disc of radius $R$. By Lemma 2.1 for $R$ sufficiently large we have

$$
\operatorname{ev}\left(\mathcal{M} \times\left(\mathbb{C} \backslash \operatorname{Int}\left(D_{R}^{2}\right)\right)\right) \subset Z \backslash W .
$$

Write $\mathcal{M}^{\prime} \subset \mathcal{M}$ for the truncated moduli space obtained by cutting off the noncompact end of $\mathcal{M}$ consisting of spheres $\{v\} \times \mathbb{C P}^{1}$ with $\psi_{V}(v)>(\log 2) / 4$. Then $\mathcal{M} \times \mathbb{C}$ strongly deformation retracts to the compact manifold (with boundary) $P:=\mathcal{M}^{\prime} \times D_{R}^{2}$.

As observed after the construction of $Z$ and $\hat{Z}$, the compact manifold $W$ (with boundary $M$ ) is a strong deformation retract of $Z$. By pre- and postcomposing ev with the respective deformation retraction, we obtain a map of pairs

$$
f:(P, \partial P) \longrightarrow(W, \partial W) \text {. }
$$

Since the degree of ev can be computed at any regular value $w$ in the interior of $W$, and neither $w$ nor the discrete set of points $\mathrm{ev}^{-1}(w) \subset P$ is affected by the deformation retractions, the map $f$ is likewise of degree 1 . This degree can now be interpreted homologically; $\operatorname{deg}(f)=1$ says that the fundamental cycle $[P] \in H_{2 n}(P, \partial P)$ is mapped to the fundamental cycle $[W] \in H_{2 n}(W, \partial W)$.

It then follows that the shriek homomorphism $f_{!}: H_{k}(W) \rightarrow H_{k}(P)$ is a right inverse for $f_{*}: H_{k}(P) \rightarrow H_{k}(W)$, since the composition $f_{*} f_{!}$is simply multiplication by the homological degree, see [4, Proposition VI.14.1].

Hence, $f_{*}: H_{k}(P) \rightarrow H_{k}(W)$ is surjective in all degrees, and the same is true for $\mathrm{ev}_{*}$, as we have merely passed to deformation retracts.

2.4. Proof of Theorem 1.2 (a). By condition (M2) we have the commutative diagram

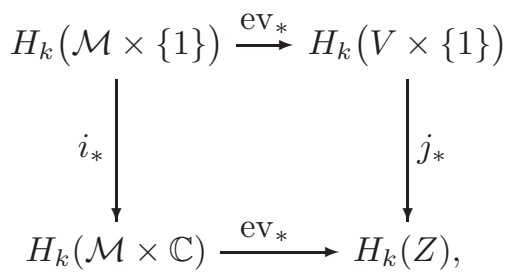

where the vertical homomorphisms are induced by inclusion. Since $i_{*}$ is an isomorphism and $\mathrm{ev}_{*}$ at the bottom is surjective by Proposition 2.4, the homomorphism $j_{*}$ is likewise surjective.

The Stein manifold $V$ has the homotopy type of an $\ell_{0}$-dimensional complex. It follows that

$$
H_{k}(Z)=0 \text { for } k \geq \ell_{0}+1 .
$$

The same homological vanishing result holds for the deformation retract $W$ of $Z$. This means that the homological dimension of $W$ can be at most that of the subcritical filling $W_{0}$. Beware that, a priori, the homotopical dimension, i.e. the smallest dimension of a $\mathrm{CW}$ complex homotopy equivalent to $W$, might well be larger.

Lemma 2.5. The relative homology group $H_{k}(W, M)$ vanishes for $k \leq 2 n-1-\ell_{0}$. 
Proof. Write $\mathrm{FH}_{*}$ and $\mathrm{TH}_{*}$ for the free and the torsion part, respectively, of a homology group $H_{*}$. By Poincaré duality and the universal coefficient theorem we have

$$
H_{k}(W, M) \cong H^{2 n-k}(W) \cong F H_{2 n-k}(W) \oplus T H_{2 n-k-1}(W) .
$$

As we have shown, the homological dimension of $W$ is at most equal to $\ell_{0}$. The lemma follows for $k<2 n-1-\ell_{0}$.

For $k=2 n-1-\ell_{0}$, it remains to show that $H_{\ell_{0}}(W)$ is a torsion-free group. Since $W_{0}$ has the homotopy type of an $\ell_{0}$-dimensional complex, the homology group $H_{\ell_{0}}\left(W_{0}\right)$ is torsion-free. From the homology exact sequence of the pair $\left(W_{0}, M\right)$ we see with Poincaré duality and the universal coefficient theorem that $H_{\ell_{0}}(M) \cong$ $H_{\ell_{0}}\left(W_{0}\right)$, so $H_{\ell_{0}}(M)$ is likewise torsion-free.

For $\ell_{0}<n-1$, the cohomology exact sequence of the pair $(W, M)$, together with the universal coefficient theorem, reduces to

$$
0 \longrightarrow H^{2 n-1-\ell_{0}}(M) \longrightarrow H^{2 n-\ell_{0}}(W, M) \longrightarrow 0,
$$

and hence $H_{\ell_{0}}(M) \cong H_{\ell_{0}}(W)$ by Poincaré duality. For $\ell_{0}=n-1$ we observe that $H_{n}(W, M) \cong T H_{n-1}(W)$ by Poincaré duality and the universal coefficient theorem, and then the relevant part of the homology exact sequence of the pair $(W, M)$ becomes

$$
0 \longrightarrow T H_{n-1}(W) \longrightarrow H_{n-1}(M) \text {. }
$$

Since $H_{n-1}(M)=H_{\ell_{0}}(M)$ is torsion-free, this implies $T H_{n-1}(W)=0$.

Remark 2.6. Alternatively, one sees from the commutative diagram that $H_{k}(W ; \mathbb{F})$ vanishes for $k \geq \ell_{0}+1$ and all fields $\mathbb{F}$. One then deduces the vanishing of the relative homology groups in the lemma over $\mathbb{F}$ with Poincaré and Kronecker duality. Since this vanishing holds for any field $\mathbb{F}$, it must also hold over $\mathbb{Z}$.

With this lemma, Theorem 1.2 (a) is an immediate consequence of the homology exact sequence of the pair $(W, M)$.

2.5. Proof of Theorem 1.2 (b). The image of $\mathcal{M} \times\{1\}$ under the evaluation map lies in $V \times\{1\}$, which we may regard as a subset of $(V \times \mathbb{C}) \backslash \operatorname{Int}\left(W_{0}\right)$. This gives us the commutative diagram

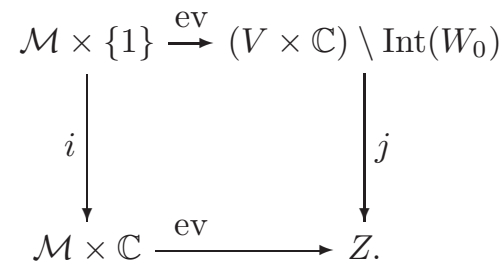

The inclusion map $i$ induces an isomorphism on fundamental groups, and the proper degree 1 map ev at the bottom, an epimorphism. The latter follows from the fact that ev: $\mathcal{M} \times \mathbb{C} \rightarrow Z$ factors through the covering of $Z$ corresponding to the characteristic subgroup $\operatorname{ev}_{*}\left(\pi_{1}(\mathcal{M} \times \mathbb{C})\right) \subset \pi_{1}(Z)$. The covering map has degree equal to the index of $\mathrm{ev}_{*}\left(\pi_{1}(\mathcal{M} \times \mathbb{C})\right)$ in $\pi_{1}(Z)$, and the degree of proper maps is multiplicative under composition.

Hence, $j$ also induces an epimorphism on fundamental groups. Up to deformation retraction, $j$ may be regarded as the inclusion map $M \subset W$. 
If $\pi_{1}(M)$ is abelian, so is its image $\pi_{1}(W)=j_{*}\left(\pi_{1}(M)\right)$, in which case these fundamental groups equal the respective first homology group. Then, by part (a) of the theorem, the inclusion $M \subset W$ is $\pi_{1}$-isomorphic.

2.6. A homology epimorphism for Liouville fillings. A close inspection of the proof of Theorem 1.2 shows that the requirement that the filling $\left(W_{0}, \omega_{0}\right)$ carry a Stein structure is not essential; the crucial point was the $\left(W_{0}, \omega_{0}\right)$ is a split manifold $V_{0} \times D^{2} \subset V \times \mathbb{C}$, where the 'end' $V \backslash V_{0}$ is of the form $\mathbb{R}_{0}^{+} \times \partial V_{0}$ and has suitable convexity properties for an analogue of Lemma 2.1 to hold.

Recall the following definitions from [9, Chapter 11].

Definition. A Liouville manifold is an exact symplectic manifold $(V, \mathrm{~d} \lambda)$ such that the corresponding Liouville vector field $Y$, defined by $i_{Y} \mathrm{~d} \lambda=\lambda$ is complete, and $V$ has an exhaustion by compact domains with smooth boundaries, along which $Y$ is outward pointing. Such compact domains in $(V, \mathrm{~d} \lambda)$ are called Liouville domains.

Let $M$ be a compact, connected manifold with a cooriented contact structure $\xi$. A compact symplectic manifold $(W, \omega)$ with boundary is called a Liouville filling of $(M, \xi)$ if $\partial W=M$ as oriented manifolds and there is a global primitive 1-form $\lambda$ for $\omega$ such that $\left.\lambda\right|_{T M}$ is a contact form for $\xi$.

Remark 2.7. Whenever we restrict attention to a compact subset of the Liouville manifold $(V, \mathrm{~d} \lambda)$ - for instance, when we consider compactly supported symplectic isotopies — we may assume without loss of generality that the Liouville manifold is of finite type, that is, it looks like the completion

$$
\left(V_{0}, \mathrm{~d} \lambda\right) \cup_{\partial V_{0}}\left(\mathbb{R}_{0}^{+} \times \partial V_{0}, \mathrm{~d}\left(\mathrm{e}^{t} \lambda_{0}\right)\right)
$$

of a Liouville domain $\left(V_{0}, \mathrm{~d} \lambda\right)$, where $\lambda_{0}:=\left.\lambda\right|_{T \partial V_{0}}$.

Theorem 2.8. Let $(M, \xi)$ be a $(2 n-1)$-dimensional compact, connected contact manifold admitting a split symplectic filling $V_{0} \times D^{2}$ (with corners rounded), where $\left(V_{0}, \mathrm{~d} \lambda\right)$ is a Liouville domain. Let $(W, \omega)$ be any symplectically aspherical filling of $(M, \xi)$. Then there is a surjective homomorphism

$$
H_{k}\left(V_{0}\right) \longrightarrow H_{k}(W)
$$

in all degrees $k \geq 0$.

If $V_{0}$ has the homotopy type of an $\ell_{0}$-dimensional complex with $\ell_{0} \leq n-1$, then the other conclusions of Theorem 1.2 hold true as well.

Proof. In the proof of Theorem 1.2 above, we now take $V$ to be the completion of $V_{0}$. In the analogue of Lemma 2.1, we work with End $:=\left(V \times \mathbb{C P}^{1}\right) \backslash\left(V_{0} \times D_{\rho}^{2}\right)$ for a disc $D_{\rho}^{2} \subset \mathbb{C} \subset \mathbb{C P}^{1}$ of suitable radius $\rho$. For (i), it is sufficient to know that $V$ is exact symplectic. For (ii) - assuming $u\left(\mathbb{C P}^{1}\right)$ intersects End but not the hypersurface $H$ - one applies the maximum principle to the $V$-component of $u$, if that component intersects $V \backslash V_{0}$, or to the $\mathbb{C}$-component, if that hits $\mathbb{C} \backslash D_{\rho}^{2}$. The proof of (iii) is analogous. Then the argument as in Section 2.4 yields the claimed homology epimorphism.

Under the additional homotopical assumption on $V_{0}$, Lemma 2.5 still holds, and then one concludes as in the proof of Theorem 1.2 


\section{Applications of Theorem 1.2}

3.1. Fillings of the standard sphere. Theorem 1.1 from the Introduction is contained in Theorem 1.2. Indeed, $\left(S^{2 n-1}, \xi_{\text {st }}\right)$ has a Stein filling given by the unit ball in $\mathbb{C}^{n}$, so we have $\ell_{0}=0$ in the notation of Theorem 1.2. This theorem then says that any other symplectically aspherical filling $(W, \omega)$ of $\left(S^{2 n-1}, \xi_{\text {st }}\right)$ is a simply connected homology ball of dimension $2 n \geq 6$, and hence diffeomorphic to the standard ball by Proposition A on page 108 of 32 .

3.2. Liouville fillings. Using relative de Rham theory, we now derive a general property of the fillings that can arise in the situation of Theorem 1.2

Proposition 3.1. Let $(M, \xi)$ be a contact manifold that admits a subcritical Stein filling. Then any symplectically aspherical filling $(W, \omega)$ of $(M, \xi)$ is a Liouville filling.

Proof. Write $i: M \rightarrow W$ for the inclusion map. The $k$ th relative de Rham chain group of the pair $(W, M)$ is given by $\Omega^{k}(i)=\Omega^{k}(W) \oplus \Omega^{k-1}(M)$, and the differential is given by $\mathrm{d}(\eta, \mu)=\left(\mathrm{d} \eta, i^{*} \eta-\mathrm{d} \mu\right)$, see [3, p. 78].

By assumption on $(W, \omega)$ being a symplectic filling of $(M, \xi)$, there is a Liouville vector field $Y$ defined near $M$ and pointing transversely outwards such that the 1-form $\lambda:=i_{Y} \omega$, defined near $M$, restricts to a contact form $\alpha:=i^{*} \lambda$ for $\xi$. We want to show that we can find a global primitive $\lambda$ with this property.

The pair $(\omega, \alpha)$ is closed, since $\mathrm{d}(\omega, \alpha)=\left(\mathrm{d} \omega, i^{*} \omega-\mathrm{d} \alpha\right)=(0,0)$, so this pair defines a class in $H_{\mathrm{dR}}^{2}(W, M) \cong H_{2 n-2}(W ; \mathbb{R})$, where as before we take the dimension of $W$ to be $2 n$. By Theorem 1.2, this last homology group vanishes, so the pair $(\omega, \alpha)$ is actually exact. This means we can find a 1 -form $\mu$ on $W$ and a smooth function $f$ on $M$ such that $(\omega, \alpha)=\left(\mathrm{d} \mu, i^{*} \mu-\mathrm{d} f\right)$.

Extend $f$ to a smooth function $F$ on $W$ and set $\lambda:=\mu-\mathrm{d} F$. Then $\mathrm{d} \lambda=\omega$ and $i^{*} \lambda=i^{*} \mu-\mathrm{d} f=\alpha$.

3.3. A result of Oancea-Viterbo. The following is Theorem 2.6 of [35].

Theorem 3.2 (Oancea-Viterbo). Let $\left(M_{1}, \xi_{1}\right)$ be a compact, connected contact manifold admitting an embedding into a subcritical Stein manifold as a hypersurface of contact type. Let $\left(W_{1}, \omega_{1}\right)$ be any symplectically aspherical filling of $\left(M_{1}, \xi_{1}\right)$ satisfying one of the following conditions:

(i) $H_{2}\left(W_{1}, M_{1}\right)=0$;

(ii) $M_{1}$ is simply connected.

Then the homomorphism

$$
H_{k}\left(M_{1}\right) \longrightarrow H_{k}\left(W_{1}\right)
$$

induced by the inclusion $M_{1} \subset W_{1}$ is surjective in all degrees $k$.

Remark 3.3. (1) The indexing 1 is used here merely to avoid notational confusion when we reprove this result below.

(2) In case (i), symplectic asphericity of any filling $\left(W_{1}, \omega_{1}\right)$ of $\left(M_{1}, \xi_{1}\right)$ is a direct consequence of the homological assumption $H_{2}\left(W_{1}, M_{1}\right)=0$, for the homomorphism $H_{2}\left(M_{1}\right) \rightarrow H_{2}\left(W_{1}\right)$ induced by inclusion is then surjective, and $\omega_{1}$ is exact near $M_{1}$.

(3) If $W_{1}$ is itself Stein (not a priori subcritical), then condition (i) is automatic for $\operatorname{dim} W_{1}=2 n \geq 6$, since $W_{1}$ then has the homotopy type of an $n$-dimensional complex, and so $H_{2}\left(W_{1}, M_{1}\right) \cong H^{2 n-2}\left(W_{1}\right)$ is zero for $2 n-2>n$. 
We now prove Theorem 3.2 As in Section 2.1 we have a contact type embedding of $\left(M_{1}, \xi_{1}\right)$ into a split Stein manifold $V \times \mathbb{C}$. Then $M_{1}$ separates $V \times \mathbb{C}$ into a compact and a non-compact component. By assumption, there is a Liouville vector field for the symplectic form on $V \times \mathbb{C}$, defined near and transverse to $M_{1}$; by [16, Theorem 3.4], this Liouville vector field points out of the compact component.

Choose a level set $M$ of the plurisubharmonic function $\psi$ on $V \times \mathbb{C}$ for a level so large that $M_{1}$ is contained in its sublevel set. Equip $M$ with the contact structure $\xi$ induced by the Stein structure. Then the compact region between $M_{1}$ and $M$ defines a symplectic cobordism $\left(W_{2}, \omega_{2}=\mathrm{d} \lambda_{2}\right)$ from $\left(M_{1}, \xi_{1}\right)$ to $(M, \xi)$.

Now, given a symplectically aspherical filling $\left(W_{1}, \omega_{1}\right)$ of $\left(M_{1}, \xi_{1}\right)$, we can glue it along this contact boundary to the cobordism $\left(W_{2}, \omega_{2}\right)$, resulting in a filling $(W, \omega)$ of the contact manifold $(M, \xi)$. The corresponding sublevel set of $\psi$ defines a subcritical Stein filling of $(M, \xi)$, so the first assumption of Theorem 1.2 is satisfied.

Lemma 3.4. Under either of the assumptions (i) or (ii) in Theorem 3.2, $(W, \omega)$ is symplectically aspherical.

Proof. Let $S$ be a 2-sphere in $W$ (i.e. a map $\phi: S^{2} \rightarrow W$, without loss of generality assumed to be smooth, with image $S$ ). We need to show that $\int_{S} \omega:=\int_{S^{2}} \phi^{*} \omega=0$. The part $\left(W_{1}, \omega_{1}\right)$ of $(W, \omega)$ is symplectically aspherical by assumption; $\left(W_{2}, \omega_{2}\right)$ is symplectically aspherical since $\omega_{2}=\mathrm{d} \lambda_{2}$ is exact. So $S$ cannot be entirely contained in only one of these two parts.

Make $S$ transverse to $M_{1}$ and write $S_{1}, S_{2}$ for the parts of $S$ contained in $W_{1}, W_{2}$, respectively.

(i) If $H_{2}\left(W_{1}, M_{1}\right)=0$, the relative cycle $S_{1}$ represents the zero class, so there is a relative 3 -chain $C$ with $\partial C=S_{1} \cup \Sigma$, where $\Sigma$ is a 2 -chain in $M_{1}$ with $\partial \Sigma=-\partial S_{1}$. It follows that

$$
\int_{S_{1}} \omega+\int_{\Sigma} \omega=\int_{\partial C} \omega=\int_{C} \mathrm{~d} \omega=0
$$

and further

$$
\int_{S_{1}} \omega=-\int_{\Sigma} \omega=-\int_{\Sigma} \mathrm{d} \lambda_{2}=-\int_{\partial \Sigma} \lambda_{2}=-\int_{\partial S_{2}} \lambda_{2}=-\int_{S_{2}} \omega_{2} .
$$

This gives $\int_{S} \omega=0$.

(ii) If $M_{1}$ is simply connected, then $S_{1}$ and $S_{2}$ can be closed off to 2 -spheres $\hat{S}_{1}, \hat{S}_{2}$ by 2 -discs in $M_{1}$. Then

$$
\int_{S} \omega=\int_{S_{1}} \omega_{1}+\int_{S_{2}} \omega_{2}=\int_{\hat{S}_{1}} \omega_{1}+\int_{\hat{S}_{2}} \omega_{2}=0
$$

by the symplectic asphericity of $\left(W_{1}, \omega_{1}\right)$ and $\left(W_{2}, \omega_{2}\right)$.

Thanks to this lemma, we can use the information from Theorem 1.2 (a) as input in the Mayer-Vietoris sequence of the decomposition $W=W_{1} \cup W_{2}$. For $k \geq n$ we have $H_{k}(W)=0$, and hence the exact sequence

$$
H_{k}\left(M_{1}\right) \longrightarrow H_{k}\left(W_{1}\right) \oplus H_{k}\left(W_{2}\right) \longrightarrow 0 ;
$$

in particular, the homomorphism $H_{k}\left(M_{1}\right) \rightarrow H_{k}\left(W_{1}\right)$ is surjective. 
For $k \leq n-1$ we still have that the homomorphism $H_{k}(M) \rightarrow H_{k}(W)$ is surjective. Consider the commutative diagram

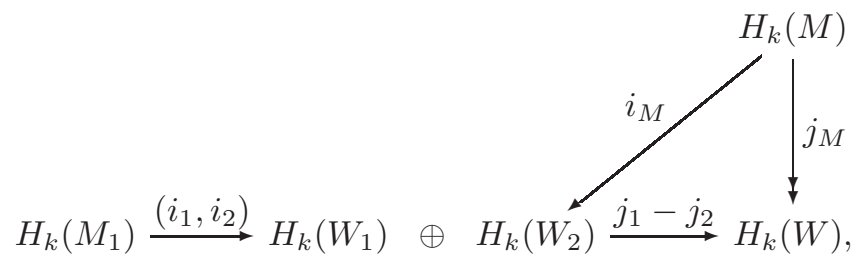

where all homomorphisms are induced by inclusion maps, the row is exact, and the vertical homomorphism is surjective. We want to show that the homomorphism $i_{1}$ is surjective. Given a class $a_{1} \in H_{k}\left(W_{1}\right)$, set $a:=j_{1}\left(a_{1}\right) \in H_{k}(W)$. Choose $A \in H_{k}(M)$ with $j_{M}(A)=a$, and set $a_{2}:=i_{M}(A) \in H_{k}\left(W_{2}\right)$, so that $j_{2}\left(a_{2}\right)=a$. It follows that $\left(a_{1}, a_{2}\right) \in H_{k}\left(W_{1}\right) \oplus H_{k}\left(W_{2}\right)$ maps to zero under $j_{1}-j_{2}$, and so this pair lies in the image of $\left(i_{1}, i_{2}\right)$. This shows that $i_{1}$ is an epimorphism, which completes the proof of Theorem 3.2 .

3.4. Extension of the Oancea-Viterbo result to $\pi_{1}$. Using Theorem 1.2 (b), we can formulate a result analogous to Theorem 3.2 for the fundamental group.

Proposition 3.5. Under the assumptions of Theorem 3.2, the normal subgroup $\mathcal{N}\left(i_{1 \#}\left(\pi_{1}\left(M_{1}\right)\right)\right)$ generated by the image of $\pi_{1}\left(M_{1}\right)$ in $\pi_{1}\left(W_{1}\right)$ equals the full group $\pi_{1}\left(W_{1}\right)$.

Proof. By Lemma 3.4 we may apply Theorem 1.2 (b) to the symplectic manifold $\left(W=W_{1} \cup W_{2}, \omega\right)$ constructed in the preceding section. Thus, we know that the homomorphism $\pi_{1}(M) \rightarrow \pi_{1}(W)$ is surjective. This homomorphism factors through $\pi_{1}\left(W_{2}\right)$, so $\pi_{1}\left(W_{2}\right) \rightarrow \pi_{1}(W)$ is likewise surjective. Here all fundamental groups are taken with a base point $* \in M$, but the last epimorphism continues to hold when we switch to a base point $*_{1} \in M_{1}$. From now on, this base point $*_{1}$ will be understood.

By Seifert-van Kampen, the fundamental group $\pi_{1}(W)$ is an amalgamated product

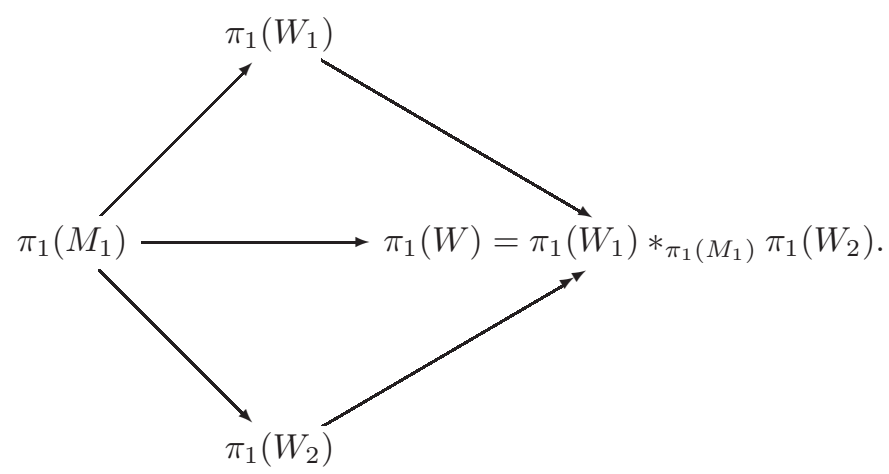

Form a $C W$ complex $W_{2}^{\prime}$ from $W_{2}$ by attaching discs to loops in $W_{1} \backslash M_{1}$ freely homotopic to a set of generators of $\pi_{1}\left(W_{2}\right)$. Then $\pi_{1}\left(W_{2}^{\prime}\right)=\{1\}$ and $W_{1} \cap W_{2}^{\prime}=$ $M_{1}$. Moreover, since the homomorphism $\pi_{1}\left(W_{2}\right) \rightarrow \pi_{1}(W)$ is surjective, the space $W_{1} \cup W_{2}^{\prime}$ is simply connected. With Seifert-van Kampen we have

$$
\{1\}=\pi_{1}\left(W_{1} \cup W_{2}^{\prime}\right)=\pi_{1}\left(W_{1}\right) *_{\pi_{1}\left(M_{1}\right)}\{1\}=\pi_{1}\left(W_{1}\right) / \mathcal{N}\left(i_{1 \#}\left(\pi_{1}\left(M_{1}\right)\right)\right) .
$$


3.5. Milnor fillable contact structures. Let $f:\left(\mathbb{C}^{n+1}, 0\right) \rightarrow(\mathbb{C}, 0), n \geq 3$, be a complex polynomial function with an isolated singularity at the origin, i.e. an isolated common zero of the partial derivatives $\partial_{z_{j}} f, j=0, \ldots, n$. Choose $\varepsilon>0$ sufficiently small so that the $\varepsilon$-disc around the origin in $\mathbb{C}^{n+1}$ does not contain any further singularities of $f$. Then the link $M_{f}$ of the singularity,

$$
M_{f}:=S_{\varepsilon}^{2 n+1} \cap\{f=0\},
$$

is a $(2 n-1)$-dimensional manifold with contact structure $\xi_{f}$ given by the complex tangencies,

$$
\xi_{f}:=T M_{f} \cap \mathrm{i}\left(T M_{f}\right),
$$

see [5]. For $\delta \in \mathbb{C}^{*}$ with $|\delta|$ sufficiently small, the smoothing

$$
W_{f}:=D_{\varepsilon}^{2 n+2} \cap\{f=\delta\}
$$

with its canonical Stein symplectic structure $\omega_{f}$ is, by Gray stability of contact structures, a Stein filling of $\left(M_{f}, \xi_{f}\right)$. We call this Stein manifold, which is unique up to deformation equivalence, the Milnor filling of $\left(M_{f}, \xi_{f}\right)$. (In [5], that name refers to the singular filling.)

The Milnor number $\mu$ is the degree of the map

$$
\begin{array}{ccc}
S_{\varepsilon}^{2 n+1} & \longrightarrow & S_{\varepsilon}^{2 n+1} \\
\mathbf{z} & \longmapsto & g(\mathbf{z}) /|g(\mathbf{z})|,
\end{array}
$$

where $g:=\left(\partial_{z_{0}} f, \ldots, \partial_{z_{n}} f\right)$. This number is always non-negative, and it equals zero precisely when the origin is actually a non-singular point of $f$.

Proposition 3.6. Suppose the contact manifold $\left(M_{f}, \xi_{f}\right)$ admits a subcritical Stein filling $\left(W_{0}, \omega_{0}\right)$. Then the following holds:

(i) $W_{0}$ and $W_{f}$ are diffeomorphic to the disc $D^{2 n}$.

(ii) The Stein structure on the Milnor filling $W_{f}$ is the standard Stein structure on the disc.

(iii) $\left(M_{f}, \xi_{f}\right)$ is contactomorphic to $\left(S^{2 n-1}, \xi_{\mathrm{st}}\right)$.

Proof. The map

$$
f /|f|: S_{\varepsilon}^{2 n+1} \backslash M_{f} \longrightarrow S^{1}
$$

is a locally trivial fibration, the closure of whose fibre (the so-called Milnor fibre) is diffeomorphic to $W_{f}$, see [34, Theorem 5.11]. Then, by [34, Theorem 6.6], which applies for $n \geq 3$, and [34, Theorem 7.2], the Milnor filling $W_{f}$ is diffeomorphic to a $2 n$-dimensional handlebody obtained by attaching $\mu$ handles of index $n$ to $D^{2 n}$; in particular, it is homotopy equivalent to a bouquet of $\mu$ spheres of dimension $n$.

Now, by Theorem 1.2 (a), the assumption on the existence of a subcritical Stein filling $\left(W_{0}, \omega_{0}\right)$ implies that $H_{k}\left(W_{f}\right)=0$ for $k \geq n$, which forces $\mu=0$. Thus, $W_{f}$ is diffeomorphic to $D^{2 n}$.

In particular, $M_{f}$ is diffeomorphic to $S^{2 n-1}$. For the argument that follows, it would be enough to know that $M_{f}$ is simply connected, which holds by 34, Theorem 5.2] and our assumption $n \geq 3$. Theorem 1.2 (b), applied to $W_{0}$, tells us that $W_{0}$ is likewise simply connected. Part (a) of the theorem, applied to both $W_{f}$ and $W_{0}$, tells us that $W_{0}$ is a homology ball. Then, as in Section 3.1, we conclude that $W_{0}$ is also diffeomorphic to $D^{2 n}$. This proves (i).

In order to prove (ii), we observe that, because of $\mu=0$, the origin is a nonsingular point of $f$. By relabelling the coordinates and multiplying $f$ by a suitable complex constant, we may assume that $\partial_{z_{0}} f(0)=1$. Then, for $\varepsilon>0$ sufficiently 
small, the linear interpolation between $f$ and the function $\mathbf{z} \mapsto z_{0}$ does not develop any singularity in $D_{\varepsilon}^{2 n}$. This interpolation provides the Stein deformation of $\left(W_{f}, \omega_{f}\right)$ to the standard Stein structure on $D^{2 n}$.

Statement (iii) is an immediate consequence of (ii).

For applications of Theorem 3.2 to Milnor fillable contact manifolds see 35, Section 6].

3.6. Distinguishing contact structures. The homological information in Theorem 1.2 gives a simple criterion to distinguish contact structures $\xi, \xi^{\prime}$ on a given manifold $M$. Suppose $(M, \xi)$ is subcritically Stein fillable with a Stein manifold of homotopical dimension $\ell_{0}$, or Liouville fillable as in Theorem 2.8 (including the homotopical assumption there), and $\left(M, \xi^{\prime}\right)$ has a symplectically aspherical filling of homotopical dimension greater than $\ell_{0}$, then $\xi$ and $\xi^{\prime}$ are not diffeomorphic.

We illustrate this with two simple examples. With $\lambda_{Q}$ we denote the canonical Liouville 1-form on the cotangent bundle of a manifold $Q$.

Example 3.7. (1) The unit sphere bundle $S\left(T^{*} S^{2} \oplus \mathbb{C}\right)$ of the stabilised cotangent bundle of $S^{2}$ is diffeomorphic to $S^{3} \times S^{2}$, and it inherits a contact structure $\xi$ from the symplectic structure $\mathrm{d} \lambda_{S^{2}}+\mathrm{d} x \wedge \mathrm{d} y$ on the unit disc bundle $D\left(T^{*} S^{2} \oplus \mathbb{C}\right)$. By [14. Example 6.2.8], we can think of $\left(S^{3} \times S^{2}, \xi\right)$ and its filling as the result of attaching a symplectic 2-handle to the standard symplectic 6-ball along a standard isotropic $S^{1} \subset\left(S^{5}, \xi_{\mathrm{st}}\right)$.

On the other hand, the standard contact structure $\xi^{\prime}$ on $S T^{*} S^{3} \cong S^{3} \times S^{2}$ with symplectically aspherical filling $\left(S^{3} \times D^{3}, \mathrm{~d} \lambda_{S^{3}}\right)$ is the result of attaching a symplectic 3-handle along a Legendrian $S^{2} \subset\left(S^{5}, \xi_{\text {st }}\right)$.

Both contact structures have vanishing first Chern class, so their underlying almost contact structures are homotopic [14, Proposition 8.1.1], but by Theorem[1.2 the contact structures are not diffeomorphic. See also [19, Example 1.9] and, for the handle descriptions, the discussion in [13, p. 1196].

(2) Likewise, the contact structures on $S^{7} \times S^{6}$ coming from the description as $S\left(T^{*} S^{6} \oplus \mathbb{C}\right)$ and $S T^{*} S^{7}$, respectively, are not diffeomorphic. This also follows from [10. Corollary 1.18], whose proof employs Rabinowitz Floer homology.

Remark 3.8. Given a (stabilised) cotangent bundle, there is a Stein structure on the unit disc bundle that provides a Stein filling of the standard contact structure on the unit sphere bundle. This follows from the explicit description of a Weinstein structure in [9, Example 11.12 (b)] and the Stein existence theorem [9, Theorem 13.5].

3.7. Fillings of unit cotangent bundles. The examples above can also be regarded as an instance of the following result.

Proposition 3.9. The unit cotangent bundle $\left(S T^{*} Q\right.$, ker $\left.\lambda_{Q}\right)$ of a closed manifold $Q$ does not admit a subcritical Stein filling.

Proof. If it did, this would produce a contradiction to Theorem 1.2, since the symplectically aspherical filling $\left(D T^{*} Q, \mathrm{~d} \lambda_{Q}\right)$ has non-trivial homology in the critical dimension.

Remark 3.10. For related results see [10, Corollary 1.18] and [1, Corollary 2.2]. 


\section{Dehn-Seidel twists}

Let $L \cong S^{n-1}$ be a Lagrangian sphere in a symplectic manifold $(V, \omega)$ of real dimension 2n-2. By the Weinstein neighbourhood theorem, cf. [29, Theorem 3.33], there is a neighbourhood of $L$ symplectomorphic to a neighbourhood of the zero section in the cotangent bundle $T^{*} S^{n-1}$ with its canonical symplectic structure $\mathrm{d} \lambda_{S^{n-1}}$. The inclusion $S^{n-1} \subset \mathbb{R}^{n}$ gives us a global coordinate description of the Liouville 1 -form $\lambda_{S^{n-1}}$. In terms of Cartesian coordinates $(\mathbf{q}, \mathbf{p}) \in \mathbb{R}^{n} \times \mathbb{R}^{n}$, the cotangent bundle $T^{*} S^{n-1} \subset \mathbb{R}^{2 n}$ is described by the equations

$$
\mathbf{q} \cdot \mathbf{q}=1 \text { and } \mathbf{q} \cdot \mathbf{p}=0
$$

then $\lambda_{S^{n-1}}=\mathbf{p}$ dq.

Define a map

$$
\tau:\left(T^{*} S^{n-1}, \mathrm{~d} \lambda_{S^{n-1}}\right) \longrightarrow\left(T^{*} S^{n-1}, \mathrm{~d} \lambda_{S^{n-1}}\right)
$$

as follows. Consider the normalised geodesic flow $\sigma_{t}$ on $T^{*} S^{n-1} \backslash S^{n-1}$ given by

$$
\sigma_{t}(\mathbf{q}, \mathbf{p})=\left(\begin{array}{cc}
\cos t & |\mathbf{p}|^{-1} \sin t \\
-|\mathbf{p}| \sin t & \cos t
\end{array}\right)\left(\begin{array}{l}
\mathbf{q} \\
\mathbf{p}
\end{array}\right) .
$$

Then set

$$
\tau(\mathbf{q}, \mathbf{p})=\sigma_{g(|\mathbf{p}|)}(\mathbf{q}, \mathbf{p}),
$$

where $r \mapsto g(r)$ is a smooth function that interpolates monotonically between $\pi$ near $r=0$ and 0 for large $r$. For $\mathbf{p}=0$ this is read as $\tau(\mathbf{q}, 0)=(-\mathbf{q}, 0)$. Then $\tau$ is a symplectomorphism of $\left(T^{*} S^{n-1}, \mathrm{~d} \lambda_{S^{n-1}}\right)$, equal to the identity for $|\mathbf{p}|$ large. Thus, $\tau$ may be regarded as a symplectomorphism of $(V, \omega)$, and it is then called a right-handed Dehn twist along $L \subset V$; for $n=2$ this coincides with the classical notion of a Dehn twist.

These generalised Dehn twists have been introduced and studied extensively by Seidel, see [38, Section 6] and [39], and they are nowadays often referred to as Dehn-Seidel twists.

Remark 4.1. For $n-1$ odd, the model Dehn twist $\tau$ on $T^{*} S^{n-1}$ is of infinite order in $\pi_{0}$ (Diff $\left.^{c}\left(T^{*} S^{n-1}\right)\right)$, where Diff ${ }^{c}$ denotes the group of compactly supported diffeomorphisms. For $n-1=2$ or 6 , the order of $\tau$ is two; for other even $n-1$ it is four or eight, see [40, p. 3311] for a discussion and references.

One example where $\tau^{2}$ is even symplectically trivial (i.e. isotopic to the identity via compactly supported symplectomorphisms) is the Dehn twist along the antidiagonal in $S^{2} \times S^{2}$ with the monotone product symplectic structure (i.e. of equal area on the two factors). Seidel proved that this example is atypical. For instance, it is shown in [39], based on the work of Gromov [20, that the group of compactly supported symplectomorphisms of $\left(T^{*} S^{2}, \mathrm{~d} \lambda_{S^{2}}\right)$ is homotopy equivalent to $\mathbb{Z}$, generated by $\tau$. Other results of [39] concern the symplectic non-triviality of $\tau^{2}$ in dimension four; in [40, Section 5] it is shown that the Dehn twist in the cotangent bundle of any higher-dimensional sphere is of infinite order symplectically. Seidel's arguments involve subtle methods from Floer homology.

We now want to prove Theorem 1.4 from the introduction, which establishes the symplectic non-triviality of any non-empty composition of right-handed Dehn twists for a broad class of symplectic manifolds, including, in particular, the cotangent bundle $\left(T^{*} S^{n-1}, \mathrm{~d} \lambda_{S^{n-1}}\right)$ per se. Thus, let $(V, \mathrm{~d} \lambda)$ be a Liouville manifold of 
dimension at least four. Write $\operatorname{Symp}^{c}(V)$ for the group of compactly supported symplectomorphisms $\phi$ of $(V, \mathrm{~d} \lambda)$.

Remark 4.2. We want to detect the symplectic non-triviality of compositions of right-handed Dehn twists in $\operatorname{Symp}^{c}(V)$. To this end, we shall be using an argument by contradiction, starting from the assumption that there is a symplectic isotopy from a given symplectomorphism of that type to the identity. This allows us, by Remark 2.7. to assume without loss of contradiction that all relevant maps and isotopies are supported in the interior of a Liouville domain $V_{0}$ whose symplectic completion is $V$.

If $\phi \in \operatorname{Symp}^{c}(V)$ is exact, i.e. $\phi^{*} \lambda-\lambda=\mathrm{d} h$ for some smooth function $h: V \rightarrow \mathbb{R}$ with compact support in $\operatorname{Int}(V)$, there is a canonical construction due to Giroux, see [14, Theorem 7.3.3] of a contact structure on the open book with page $\operatorname{Int}\left(V_{0}\right)$ and monodromy $\phi$. We denote this contact manifold by $\operatorname{Open}\left(V_{0}, \phi\right)$. We want to show that if $\phi$ lies in the identity component $\operatorname{Symp}_{0}^{c}(V)$ of $\operatorname{Symp}^{c}(V)$, i.e. if $\phi$ is isotopic to the identity via compactly supported but not, a priori, exact symplectomorphisms, then the resulting contact manifold does not depend, up to contactomorphism, on the specific choice of such $\phi$.

Proposition 4.3. If $\phi \in \operatorname{Symp}_{0}^{c}(V)$ is exact, then $\operatorname{Open}\left(V_{0}, \phi\right)$ is contactomorphic to $\operatorname{Open}\left(V_{0}\right.$, id).

Proof. As shown in [29, Chapter 10], there is an exact sequence

$$
\begin{aligned}
& 0 \longrightarrow \operatorname{Ham}^{c}(V) \longrightarrow \operatorname{Symp}_{0}^{c}(V) \longrightarrow H_{c}^{1}(V ; \mathbb{R}) \longrightarrow 0, \\
& \phi \quad \longmapsto\left[\phi^{*} \lambda-\lambda\right]
\end{aligned}
$$

where $\operatorname{Ham}^{c}(V)$ denotes the group of compactly supported Hamiltonian diffeomorphisms, that is, symplectomorphisms that are the time-1 map of a Hamiltonian isotopy with support in a compact subset (depending on the isotopy), and $H_{c}^{1}(V ; \mathbb{R})$ denotes the compactly supported de Rham cohomology of $V$.

Thus, our assumptions imply that $\phi$ is actually Hamiltonian. Let $\left(H_{t}\right)_{t \in[0,1]}$ be the time-dependent Hamiltonian function generating the Hamiltonian isotopy $\left(\phi_{t}\right)_{t \in[0,1]}$ with $\phi_{0}=$ id and $\phi_{1}=\phi$. Write $X_{t}$ for the corresponding time-dependent Hamiltonian vector field. Then

$$
\phi_{t}^{*} \lambda-\lambda=\mathrm{d} h_{t},
$$

where

$$
h_{t}:=\int_{0}^{t} \phi_{s}^{*}\left(\lambda\left(X_{s}\right)-H_{s}\right) \mathrm{d} s,
$$

see [29, Proposition 9.19].

With (11) one deduces $\operatorname{Open}(V$, id $) \cong \operatorname{Open}(V, \phi)$ from the explicit construction of the contact open book in [14, Theorem 7.3.3], using Gray stability.

The following lemma will allow us to apply this observation to compositions of Dehn twists. Here the assumption $\operatorname{dim} V \geq 4$ is used.

Lemma 4.4. Any composition of Dehn twists on $(V, \mathrm{~d} \lambda)$ is an exact symplectomorphism.

Proof. For the model Dehn twist $\tau$ on $\left(T^{*} S^{n-1}, \mathrm{~d} \lambda_{S^{n-1}}\right)$, an explicit function $h$ with $\tau^{*} \lambda_{S^{n-1}}-\lambda_{S^{n-1}}=\mathrm{d} h$ is described in 24. The choice of primitive $\lambda_{S^{n-1}}$ for 
the symplectic form, however, is irrelevant for the exactness of $\tau$, as follows from a simple homological consideration.

If $(U, \mathrm{~d} \mu)$ is any open $(2 n-2)$-dimensional symplectic manifold (without boundary) with $H^{2 n-3}(U ; \mathbb{R})=0$, such as a tubular neighbourhood of a Lagrangian sphere (for $n \geq 3$ ), then $H_{c}^{1}(U ; \mathbb{R})=0$ by Poincaré duality for compactly supported cohomology [3, p. 44]. Then, any compactly supported symplectomorphism of $(U, \mathrm{~d} \mu)$ is exact, regardless of the choice of primitive $\mu$ for the symplectic form. Hence, this remains true if $(U, \mathrm{~d} \mu)$ admits a (not necessarily exact!) symplectic embedding into a larger symplectic manifold $(V, \mathrm{~d} \lambda)$, and the symplectomorphism is regarded as an automorphism of $V$. In particular, Dehn twists on exact symplectic manifolds of dimension $2 n-2 \geq 4$ are always exact.

A straightforward calculation shows that the composition of exact symplectomorphisms is likewise exact.

Proof of Theorem 1.4. Arguing by contradiction, we assume that $\phi$ is a non-trivial composition of right-handed Dehn twists on the Liouville manifold $(V, \mathrm{~d} \lambda)$ which is symplectically isotopic to the identity, i.e. contained in $\operatorname{Symp}_{0}^{c}(V)$. By Lemma 4.4 and Proposition 4.3 the contact open book $\operatorname{Open}\left(V_{0}, \phi^{N}\right)$ is contactomorphic to $\operatorname{Open}\left(V_{0}\right.$, id $)$ for any natural number $N \in \mathbb{N}$.

The contact open book $(M, \xi):=\operatorname{Open}\left(V_{0}\right.$, id $)$ is symplectically filled by $V_{0} \times D^{2}$ (after rounding corners inside $V \times \mathbb{C}$ ). This places us in the situation of Theorem 2.8.

Now we use the specific nature of $\phi$ as a composition of right-handed Dehn twists. By 23. Lemma 4.2], a Lagrangian sphere $L$ in the page of a contact open book may be assumed to be Legendrian in the open book. Then, by [23, Theorem 4.4], composing the monodromy of the given open book with the right-handed Dehn twist along $L$ is equivalent to performing a Weinstein surgery on the open book along $L$; see [15] for a simpler description in the 3-dimensional situation.

This gives us a Liouville (and hence symplectically aspherical) filling $\left(W_{N}, \omega_{N}\right)$ of $\operatorname{Open}\left(V_{0}, \phi^{N}\right) \cong(M, \xi)$ for any $N \in \mathbb{N}$. By Theorem 2.8 we have, in particular, a homology epimorphism $H_{n}\left(V_{0}\right) \rightarrow H_{n}\left(W_{N}\right)$.

On the other hand, $W_{N}$ is obtained from $V_{0} \times D^{2}$ by attaching $k N$ handles of index $n$, where $k$ is the number of Dehn twists in the composition $\phi$. Think of $W_{N}$ as decomposed into $V_{0} \times D^{2}$ and the handles, with intersection given by the disjoint neighbourhoods, each diffeomorphic to $S^{n-1} \times D^{n}$, of $k N$ attaching spheres in $\partial\left(V_{0} \times D^{2}\right)$. The relevant part of the Mayer-Vietoris sequence,

$$
H_{n}\left(W_{N}\right) \longrightarrow H_{n-1}\left(\sqcup_{k N} S^{n-1}\right) \longrightarrow H_{n-1}\left(V_{0}\right),
$$

gives us the estimate

$$
b_{n}\left(W_{N}\right)+b_{n-1}\left(V_{0}\right) \geq k N
$$

on Betti numbers. Together with the epimorphism $H_{n}\left(V_{0}\right) \rightarrow H_{n}\left(W_{N}\right)$ we have

$$
b_{n}\left(V_{0}\right)+b_{n-1}\left(V_{0}\right) \geq k N
$$

for all $N \in \mathbb{N}$, which is a contradiction.

Remark 4.5. With the notation from this section, Theorem 1.4 can be rephrased as saying that for any non-trivial composition $\phi$ of right-handed Dehn twists on a Liouville manifold $(V, \mathrm{~d} \lambda)$, the class of $\phi$ is of infinite order in $\pi_{0}\left(\operatorname{Symp}^{c}(V)\right)$.

Remark 4.6. For the iteration of a single Dehn twist on a Liouville domain, Theorem 1.4 has been proved by Uljarević [43, Corollary 5.6.3], using Floer-theoretic 
methods. The analogue of the theorem for iterations of a single fibred or fractional Dehn twist, under various technical assumptions, are proved in [6, Theorem B], 7, Corollary 1.2] and [42, Corollary 1.4]. The papers [6, 7] also build on the idea to use the iterated (fractional or fibred) Dehn twist as the monodromy of an open book. The infinite order of the Dehn twist is then established by considering the mean Euler characteristic in symplectic homology, or by studying filling obstructions.

\section{Proof of Theorem 1.5}

Let $(M, \xi)$ be a simply connected contact manifold of dimension at least five, with a subcritical Stein filling $\left(W_{0}, \omega_{0}\right)$, and let $(W, \omega)$ be any other symplectically aspherical filling of $(M, \xi)$. Our aim is to show that $W$ must be diffeomorphic to $W_{0}$.

As in the proof of Theorem 1.2, we may think of $W$ as a level set of a plurisubharmonic potential on a split Stein manifold $V \times \mathbb{C}$, and of $W_{0}$ as the corresponding sublevel set.

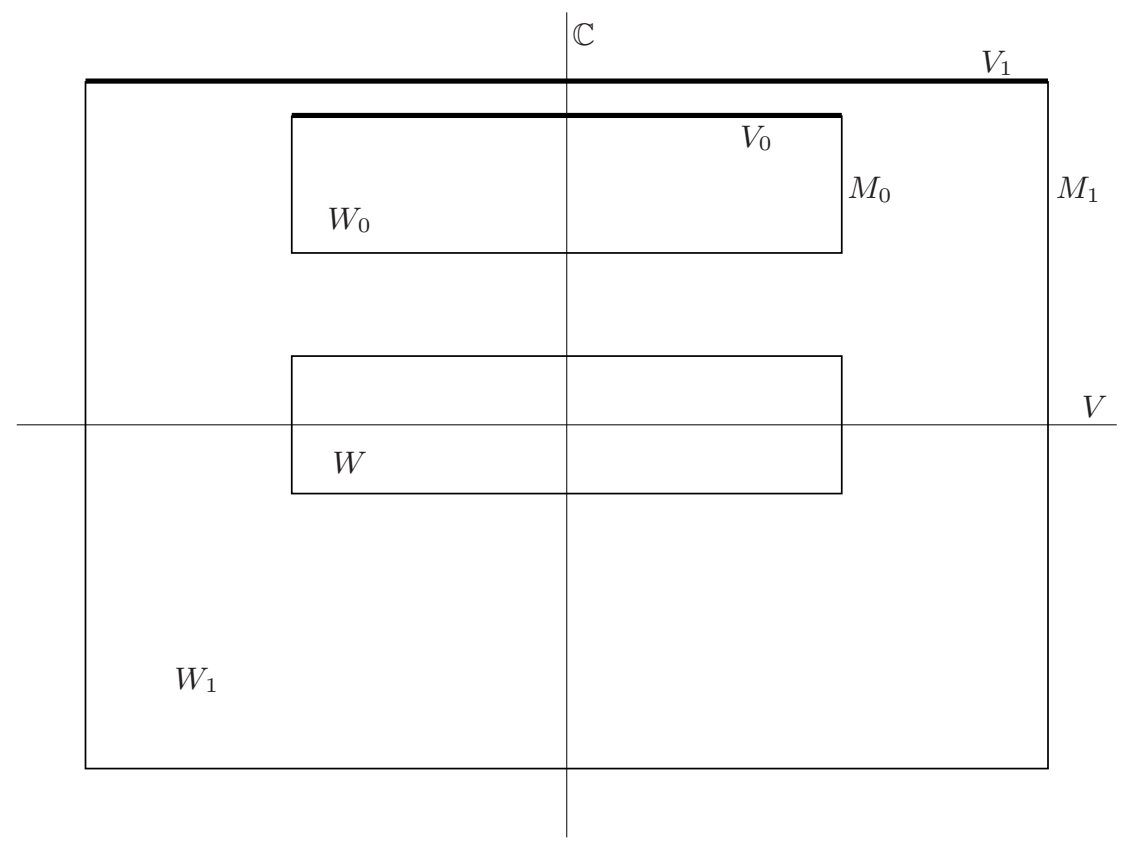

Figure 2. The cobordism $X=W_{1} \backslash \operatorname{Int}\left(W_{0}\right)$.

Consider the schematic picture shown in Figure 2, From now on, the argument is essentially topological. This allows us to think of $W_{0}$ as $V_{0} \times D^{2}$, where $V_{0}$ is a Stein domain with symplectic completion $V$ (in the sense of Remark 2.7).

We build a manifold $W_{1}$ as follows. Add a (sufficiently large) collar neighbourhood to $W_{0}$, i.e. pass to a higher sublevel set of the plurisubharmonic potential, with boundary $M_{1} \cong M$. There is a topological copy of $W_{0}$ inside this neighbourhood (and it is this which is shown in Figure 2), disjoint from the original one, simply given by translation in the $\mathbb{C}$-direction. Cut out the original copy of $W_{0}$ and replace it by $W$; this can be done symplectically. The resulting symplectic manifold 
$W_{1}$ with boundary $M_{1} \cong M$ is simply $W$ with a collar added, and Theorem 1.2 applies to it.

Inside the boundary $M_{0} \cong M$ of $W_{0}$, there is a copy of $V_{0}$, which we can think of as $V_{0} \times\{1\} \subset V_{0} \times D^{2}=W_{0}$. There is a corresponding copy $V_{1}$ of $V_{0}$ inside $M_{1}$. Apart from the inclusion $W_{0} \rightarrow W_{1}$, there is a second embedding $W_{0} \rightarrow W_{1}$ that maps $V_{0}$ diffeomorphically to $V_{1}$, obtained by extending an isotopy that moves $V_{0}$ to $V_{1}$.

Now set $X=W_{1} \backslash \operatorname{Int}\left(W_{0}\right)$, which is a cobordism between $M_{0}$ and $M_{1}$. This gives us the following diagram of maps. We shall refer to it in the sequel as the 'cobordism diagram'.

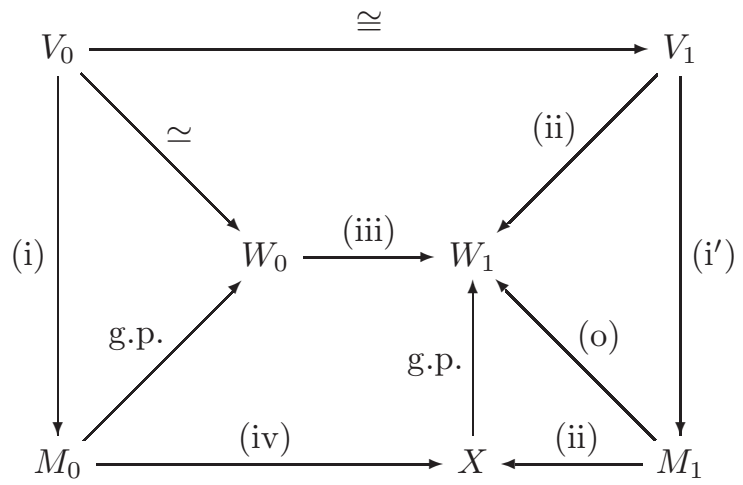

All maps in this diagram are inclusions, except for $V_{0} \rightarrow V_{1}$, which is the diffeomorphism just mentioned, and $W_{0} \rightarrow W_{1}$, which indicates both the inclusion and the alternative embedding just described. With this understood, the cobordism diagram is homotopy commutative.

By Theorem 1.2 the inclusion $M_{1} \rightarrow W_{1}$, which is essentially the inclusion $M \rightarrow$ $W$, is always $\pi_{1}$-surjective. Under the assumption that $M$ is simply connected, it is of course also $\pi_{1}$-injective. Since, later on, we shall be considering more general situations, we formulate the next result in terms of this assumption.

Lemma 5.1. If the inclusion $M \rightarrow W$ is $\pi_{1}$-injective, then the inclusion maps $M_{0}, M_{1} \rightarrow X$ are $\pi_{1}$-isomorphic.

Proof. The following steps refer to the maps with the corresponding labels in the cobordism diagram.

(g.p.) This label stands for 'general position'. First consider the inclusion $M_{0} \rightarrow$ $W_{0}$. By assumption, $W_{0}$ has a handle decomposition with handles of index at most $n-1$. This allows us to define a Morse-Smale function on $W_{0}$ whose negative gradient flow contracts $W_{0}$ onto a subcomplex of dimension at most $n$, which we call the skeleton. (Notice that the dimension of the skeleton may be larger than the homotopical dimension of $W_{0}$. We do not care about homologically inessential handles, as long as they have subcritical index.) Under the positive gradient flow, the complement of the skeleton flows into the boundary $M_{0}$.

Now consider a relative $k$-disc in $W_{0}$, that is, a continuous map $\left(D^{k}, S^{k-1}\right) \rightarrow$ $\left(W_{0}, M_{0}\right)$. By general position, we can make this disc (rel boundary) disjoint from the skeleton, provided that $k+n-1<2 n$. The gradient flow of the Morse-Smale function then allows us to push that disc (rel boundary) into $M_{0}$. 
For $k \in\{1,2\}$, that inequality is satisfied for all $n \geq 2$. It follows that the relative homotopy groups $\pi_{1}\left(W_{0}, M_{0}\right)$ and $\pi_{2}\left(W_{0}, M_{0}\right)$ are trivial, which implies that the inclusion $M_{0} \rightarrow W_{0}$ is $\pi_{1}$-isomorphic.

In an analogous fashion, we can deal with the inclusion $X \rightarrow W_{1}$. Given a relative $k$-disc $\left(D^{k}, S^{k-1}\right) \rightarrow\left(W_{1}, X\right)$, for $k \in\{1,2\}$ we can again make it disjoint from the skeleton of $W_{0}$, and then use the gradient flow to push it into $X$.

(o) The map $M_{1} \rightarrow W_{1}$ is $\pi_{1}$-isomorphic by Theorem 1.2 and our assumption.

(i) The inclusion $V_{0} \rightarrow M_{0}$ is $\pi_{1}$-isomorphic, since both the homotopy equivalence $V_{0} \rightarrow W_{0}$ and the inclusion $M_{0} \rightarrow W_{0}$ have this property.

(i') The inclusion $V_{1} \rightarrow M_{1}$ is then likewise $\pi_{1}$-isomorphic.

(ii) With (i') it follows that $V_{1} \rightarrow W_{1}$ is $\pi_{1}$-isomorphic, and the same is true for $M_{1} \rightarrow X$ by (g.p.).

(iii) Interpreting $W_{0} \rightarrow W_{1}$ as the 'alternative embedding', we conclude from (ii) that this map is $\pi_{1}$-isomorphic.

(iv) It now follows that $M_{0} \rightarrow X$ is $\pi_{1}$-isomorphic.

Next we analyse the maps in the cobordism diagram with a view to homology. The following lemma does not presuppose any information on the fundamental group of $M$. The input previously provided by a general position argument or by the assumption on $\pi_{1}$-injectivity now comes directly from Theorem 1.2 .

Lemma 5.2. The relative homology groups $H_{k}\left(X, M_{0}\right)$ and $H_{k}\left(X, M_{1}\right)$ vanish for all $k \in \mathbb{N}_{0}$.

Proof. By Theorem 1.2, the inclusions $M_{0} \rightarrow W_{0}$ and $M_{1} \rightarrow W_{1}$ induce isomorphisms on $H_{k}$ for $k=0, \ldots, \ell_{0}$.

(i), (i') It follows that the inclusion $V_{0} \rightarrow M_{0}$ induces isomorphisms in homology up to degree $\ell_{0}$, and hence so does the inclusion $V_{1} \rightarrow M_{1}$.

(ii) The same is then true for the inclusion $V_{1} \rightarrow W_{1}$.

(iii) Since the homology groups of $W_{0}$ and $W_{1}$ in degree $k>\ell_{0}$ are trivial by Theorem 1.2, we conclude that the inclusion $W_{0} \rightarrow W_{1}$ induces an isomorphism in homology, and hence $H_{k}\left(W_{1}, W_{0}\right)=0$ for all $k$.

(iv) By excision we have $H_{k}\left(X, M_{0}\right) \cong H_{k}\left(W_{1}, W_{0}\right)=0$. With Poincaré duality and the universal coefficient theorem we conclude $H_{k}\left(X, M_{1}\right)=0$.

Proof of Theorem 1.5. By Lemmata 5.1 and 5.2 and the relative Hurewicz theorem, the simply connected cobordism $\left\{M_{0}, X, M_{1}\right\}$ is an $h$-cobordism. Hence, as $n \geq 3$, it is diffeomorphic to a product $[0,1] \times M$ by the $h$-cobordism theorem. It follows that $W$, which is diffeomorphic to $W_{1}$, is obtained from $W_{0}$ by attaching this collar $[0,1] \times M$, so $W$ and $W_{0}$ are diffeomorphic.

A closer inspection of the argument in this section immediately yields the following generalisation of Theorem 1.5. For a classical survey on Whitehead groups and Whitehead torsion see 33 .

Theorem 5.3. Let $(M, \xi)$ be a manifold as in Theorem 1.2 with $\pi_{1}(M)$ finite. Then the symplectically aspherical fillings $(W, \omega)$ of $(M, \xi)$ for which the inclusion $M \rightarrow$ $W$ is $\pi_{1}$-injective (which by Theorem 1.2 are all symplectically aspherical fillings when $\pi_{1}(M)$ is finite abelian) are pairwise homotopy equivalent. If, in addition, $n \geq$ 3 and the fundamental group $\pi_{1}(M)$ has trivial Whitehead group $\mathrm{Wh}\left(\pi_{1}(M)\right)=0$, then these fillings are pairwise diffeomorphic. 
Proof. As observed in the proof of Lemma 5.1 the inclusion $M \rightarrow W_{0}$ into the subcritical Stein filling is always $\pi_{1}$-isomorphic by a general position argument. The same is true for the inclusion $M \rightarrow W$ by assumption and Lemma 5.1. It follows that the (compact!) universal cover of $(M, \xi)$ is filled by either of the universal covers of $\left(W_{0}, \omega_{0}\right)$ and $(W, \omega)$.

As before, we now investigate the cobordism $\left\{M_{0}, X, M_{1}\right\}$. Again by Lemma 5.1 here too we can pass to the compact cobordism $\left\{\widetilde{M}_{0}, \widetilde{X}, \widetilde{M}_{1}\right\}$ of universal covers. Our previous argument shows that this is an $h$-cobordism, hence so is $\left\{M_{0}, X, M_{1}\right\}$. It follows that $W_{1}$, which is a diffeomorphic copy of $W$, is homotopy equivalent to $W_{0}$, since $W_{1}=W_{0} \cup_{M_{0}}\left\{M_{0}, X, M_{1}\right\}$.

Under the assumption $\operatorname{Wh}\left(\pi_{1}(M)\right)=0$, the cobordism $\left\{M_{0}, X, M_{1}\right\}$ is an $s$ cobordism, and hence trivial for $n \geq 3$.

\section{Coverings}

Starting from a symplectically aspherical filling $(W, \omega)$ of $(M, \xi)$ as in Theorem 1.2, we now analyse the argument for a covering $W^{\prime} \rightarrow W$. This covering is not assumed to be finite, so it includes the case of the universal covering $\widetilde{W} \rightarrow W$ when $\pi_{1}(W)$ has infinite order. Our main applications will concern the situation when the universal cover $\widetilde{W}_{0}$ of the presumed subcritical Stein filling $\left(W_{0}, \omega_{0}\right)$ of $(M, \xi)$ is contractible.

We shall assume throughout that $W^{\prime}$ is connected. The covering $W^{\prime} \rightarrow W$ induces a covering $\partial W^{\prime}=: M^{\prime} \rightarrow M$. Since, by Theorem 1.2 (b), the inclusion $M \rightarrow W$ is $\pi_{1}$-surjective, the manifold $M^{\prime}$ must likewise be connected, as is seen by a standard covering space argument.

As observed in the proof of Lemma 5.1, the inclusion $M \rightarrow W_{0}$ is $\pi_{1}$-isomorphic thanks to $W_{0}$ being contractible onto its skeleton of dimension at most $n-1$. So there is a covering $W_{0}^{\prime} \rightarrow W_{0}$ inducing the covering $M^{\prime} \rightarrow M$ on the boundary, and a corresponding covering $V^{\prime} \rightarrow V$ in the notation of Section 2.1. Given this information, we can then define symplectic manifolds $\left(Z^{\prime}, \Omega^{\prime}\right)$ and $\left(\hat{Z}^{\prime}, \hat{\Omega}^{\prime}\right)$ in complete analogy with the construction in that section. Write $J^{\prime}$ for the lifted almost complex structure on $\hat{Z}^{\prime}$. Then $J^{\prime}$ is uniformly tamed by $\hat{\Omega}^{\prime}$, and the metric $g^{\prime}:=\hat{\Omega}^{\prime}\left(., J^{\prime}\right.$.) is complete and admits both an upper bound on the sectional curvature and a positive lower bound on the injectivity radius, that is, $\left(\hat{Z}^{\prime}, \hat{\Omega}^{\prime}\right)$ is geometrically bounded in the sense of [2, Definition 2.2.1].

As before, we define a moduli space $\mathcal{M}^{\prime}$ of holomorphic spheres $u^{\prime}: \mathbb{C P}^{1} \rightarrow$ $\left(\hat{Z}^{\prime}, J^{\prime}\right)$ subject to the analogous conditions (M1) and (M2). The composition of such holomorphic spheres with the covering map $\mathfrak{p}: \hat{Z}^{\prime} \rightarrow \hat{Z}$ defines a covering $\mathcal{M}^{\prime} \rightarrow \mathcal{M}$.

Proposition 2.3 holds unchanged; we need only establish properness of the evaluation map in the new setting.

Lemma 6.1. The evaluation map

$$
\begin{aligned}
\mathrm{ev}^{\prime}: \quad \mathcal{M}^{\prime} \times \mathbb{C P}^{1} & \longrightarrow \hat{Z}^{\prime} \\
\left(u^{\prime}, z\right) & \longmapsto u^{\prime}(z)
\end{aligned}
$$

is proper. 
Proof. Given a compact subset $K \subset \hat{Z}^{\prime}$, consider a sequence $\left(u_{\nu}^{\prime}, z_{\nu}\right)$ in $\left(\mathrm{ev}^{\prime}\right)^{-1}(K)$. We may assume that $z_{\nu} \rightarrow z_{0} \in \mathbb{C P}^{1}$ and $u_{\nu}^{\prime}\left(z_{\nu}\right) \rightarrow p_{0}^{\prime} \in K$ for $\nu \rightarrow \infty$. By Proposition 2.3. we may further assume that the sequence $u_{\nu}:=\mathfrak{p} \circ u_{\nu}^{\prime}$ of holomorphic curves in $\hat{Z}$ is $C^{\infty}$-convergent.

For a given $w \in \mathbb{C P}^{1}$, let $\gamma_{\nu}$ be a unit speed geodesic in $\mathbb{C P}^{1}$ of length $L_{\nu} \leq \pi / 2$ with respect to the Fubini-Study metric, connecting $w$ with $z_{\nu}$. Then, thanks to $\left(\hat{Z}^{\prime}, \hat{\Omega}^{\prime}\right)$ being geometrically bounded, the distance between $u_{\nu}^{\prime}(w)$ and $u_{\nu}^{\prime}\left(z_{\nu}\right)$ in $\hat{Z}^{\prime}$ with respect to the metric $g^{\prime}$ can be estimated from above by

$$
\operatorname{dist}\left(\left(u_{\nu}^{\prime}(w), u_{\nu}^{\prime}\left(z_{\nu}\right)\right) \leq \int_{0}^{L_{\nu}}\left|\frac{\mathrm{d}}{\mathrm{d} t}\left(u_{\nu}^{\prime} \circ \gamma_{\nu}\right)\right| \mathrm{d} t \leq \text { const. } \cdot\left\|T u_{\nu}\right\|_{C^{0}} \leq \text { const. },\right.
$$

with constants that do not depend on $w$. Hence, the images $u_{\nu}^{\prime}\left(\mathbb{C P}^{1}\right)$ are all contained in a sufficiently large closed metric ball about $p_{0}^{\prime}$, which is a compact subset of the complete Riemannian manifold $\left(\hat{Z}^{\prime}, g^{\prime}\right)$.

This guarantees the existence of a Gromov-convergent subsequence of $\left(u_{\nu}^{\prime}\right)$. The argument then concludes as in the proof of Proposition 2.3.

The arguments in Sections 2.3 and 2.4 then go through, mutatis mutandis, for the covering spaces, with the proviso that cohomology be replaced by cohomology with compact supports in all arguments requiring (implicitly or explicitly) Poincaré duality. See [21, pp. 242-249] for a good exposition of Poincaré duality in this context.

In particular, again we obtain a homology epimorphism $H_{k}\left(V^{\prime}\right) \rightarrow H_{k}\left(W^{\prime}\right)$ for all $k$. The following proposition is the simplest consequence of this fact, but one that has wide-ranging applications, as we shall see.

Proposition 6.2. With $M, W_{0}, W$ as in Theorem 1.2, suppose that the inclusion $M \rightarrow W$ is $\pi_{1}$-injective and $\widetilde{W}_{0}$ is contractible. Then $\widetilde{W}$ is likewise contractible.

Proof. Under the assumption that the inclusion $M \rightarrow W$ is $\pi_{1}$-injective and hence, by Theorem 1.2 (b), $\pi_{1}$-isomorphic, the universal covering $\widetilde{W} \rightarrow W$ restricts to the universal covering $\widetilde{M} \rightarrow M$ on the boundary. The assumption on $\widetilde{W}_{0}$ being contractible is the same as saying that the universal cover $\widetilde{V}$ is contractible. The mentioned homology epimorphism then implies that $\widetilde{W}$ is a simply connected space with vanishing reduced homology, and hence contractible.

In Section 8 we shall make use of the following lemma.

Lemma 6.3. With $M, W_{0}, W$ as in Theorem 1.2. suppose that the inclusion $M \rightarrow$ $W$ is $\pi_{1}$-injective. Then the inclusion $\widetilde{M}_{0} \rightarrow \widetilde{X}$ induces a surjective homomorphism in homology.

Proof. The assumption on $\pi_{1}$ allows us to pass to universal covers in the cobordism diagram in Section 5 . Consider the following commutative diagram with exact rows:

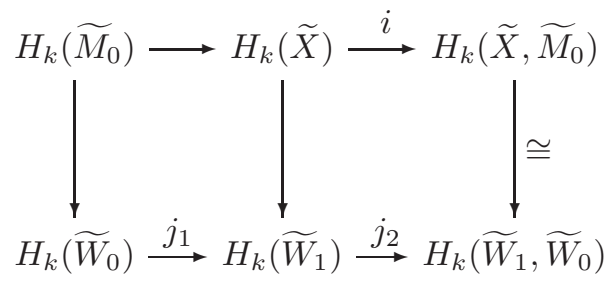


The vertical map on the right is the excision isomorphism. The homology epimorphism $H_{k}\left(V^{\prime}\right) \rightarrow H_{k}\left(W^{\prime}\right)$ for any covering gives us, in particular, an epimorphism $H_{k}\left(\widetilde{V}_{1}\right) \rightarrow H_{k}\left(\widetilde{W}_{1}\right)$. From the cobordism diagram we then see that $j_{1}$ is likewise surjective, and hence $j_{2}$ the zero homomorphism. This in turn implies that $i$ is the zero homomorphism.

\section{Handle Decompositions}

In this section we discuss the homotopy and diffeomorphism classification of fillings of a given closed, connected contact manifold $(M, \xi)$ of dimension $2 n-1$ under the assumption that information is given on the maximal index in a handle decomposition of the filling. Applications include the homotopy classification of subcritical Stein fillings.

Thus, let $M, W, W_{0}$ be as in Theorem 1.2. with the additional assumption that $W$ has a handle decomposition involving handles of index $\leq \ell$ only. Then a general position argument as in the proof of Lemma 5.1 yields the following result.

Lemma 7.1. For $k \leq 2 n-1-\ell_{0}$, the relative groups $\pi_{k}\left(W_{0}, M\right)$ and $H_{k}\left(W_{0}, M\right)$ are trivial. The same is true for the relative groups $\pi_{k}\left(W_{1}, X\right)$ and $H_{k}\left(W_{1}, X\right)$. For $k \leq 2 n-1-\ell$, the relative groups $\pi_{k}(W, M)$ and $H_{k}(W, M)$ are trivial.

In particular, for $\ell \leq 2 n-3$ the inclusion $M \rightarrow W$ is $\pi_{1}$-isomorphic. Then, by the proof of Lemma 5.1 the same will be true for all the other maps in the

cobordism diagram, so that we can pass simultaneously to the universal covers of all spaces in that diagram. From now on, this assumption will be understood.

Theorem 7.2. If $\ell_{0}+\max \left(\ell_{0}, \ell\right) \leq 2 n-2$, then $W$ and $W_{0}$ are homotopy equivalent.

Proof. From the lemma it follows that all maps in the cobordism diagram, also at the level of universal covers, are $\pi_{k^{-}}$and $H_{k}$-isomorphic for $k \leq 2 n-2-\max \left(\ell_{0}, \ell\right)$. By the assumption in the theorem, this holds in particular for $k \leq \ell_{0}$.

This implies that the inclusion $\widetilde{W}_{0} \rightarrow \widetilde{W}_{1}$ induces an isomorphism in homology in all degrees, since

$$
H_{k}\left(\widetilde{W}_{0}\right)=0=H_{k}\left(\widetilde{W}_{1}\right) \text { for } k \geq \ell_{0}+1
$$

for $\widetilde{W}_{0}$ this follows from the homotopical assumptions; for $\widetilde{W}_{1} \cong \widetilde{W}$, from the homology epimorphism in Section 6 .

The homology exact sequence of the pair $\left(\widetilde{W}_{1}, \widetilde{W}_{0}\right)$ then shows the vanishing of $H_{k}\left(\widetilde{W}_{1}, \widetilde{W}_{0}\right)$ in all degrees. By excision, we also have $H_{k}\left(\widetilde{X}, \widetilde{M}_{0}\right)=0$ for all $k$.

With the relative Hurewicz theorem we deduce $\pi_{k}\left(\tilde{X}, \widetilde{M}_{0}\right)=0$ for all $k$. Since the inclusion $M_{0} \rightarrow X$ is already known to be a $\pi_{1}$-isomorphism, $M_{0}$ is a strong deformation retract of $X$ by Whitehead's theorem [21, Theorem 4.5]. Hence

$$
W \simeq W_{1}=W_{0} \cup_{M_{0}} X \simeq W_{0},
$$

as we wanted to show.

The proof of the result that all subcritical Stein fillings of a given contact manifold are homotopy equivalent is now straightforward.

Proof of Theorem 1.6. For a subcritical filling we have $\ell \leq n-1$, and hence $\ell_{0}+$ $\max \left(\ell_{0}, \ell\right) \leq 2 n-2$. 
The required estimate is also satisfied for $\ell \leq n$, provided that $\ell_{0} \leq n-2$. This gives the following corollary.

Corollary 7.3. If $(M, \xi)$ admits a subcritical Stein filling with $\ell_{0} \leq n-2$, then all Stein fillings (including critical ones) are homotopy equivalent.

\section{Simple SPACES}

In this section we prove Theorem 1.7. Thus, consider manifolds $M, W_{0}, W$ as in Theorem 1.2 under the additional assumption that $M$ is a simple space and $n \geq 3$. In particular, since the action of $\pi_{1}$ on itself is given by conjugation, $\pi_{1}(M)$ must be abelian. Thus, by Theorem 1.2 (b) and Lemma 5.1, all maps in the cobordism diagram are $\pi_{1}$-isomorphic, and we can pass to universal covers. Also, from Lemma 5.2 we know that the relative homology groups $H_{k}\left(X, M_{0}\right)$ and $H_{k}\left(X, M_{1}\right)$ vanish for all $k$.

The assumptions of Theorem 1.7 are taken for granted in this section.

Lemma 8.1. The relative homotopy groups $\pi_{k}\left(X, M_{0}\right)$ are trivial.

Proof. The statement holds for $k=0,1$, since $M_{0}$ and $X$ are connected, and the inclusion map $M_{0} \rightarrow X$ is $\pi_{1}$-isomorphic.

Inductively, we assume that the vanishing of $\pi_{i}\left(X, M_{0}\right)$ has been established for $i \leq k-1$. We want to show $\pi_{k}\left(X, M_{0}\right)=0$.

Write $\gamma(\eta) \in \pi_{k}\left(X, M_{0}\right)$ for the element obtained by the action of $\gamma \in \pi_{1}\left(M_{0}\right)$ on $\eta \in \pi_{k}\left(X, M_{0}\right)$. By the relative Hurewicz theorem, the Hurewicz homomorphism

$$
h_{k}: \pi_{k}\left(X, M_{0}\right) \longrightarrow H_{k}\left(X, M_{0}\right)
$$

is an epimorphism whose kernel is the subgroup of $\pi_{k}\left(X, M_{0}\right)$ generated by elements of the form $\gamma(\eta)-\eta$. Notice that the inclusion $M_{0} \rightarrow X$ being $\pi_{1}$-isomorphic implies that $\pi_{2}\left(X, M_{0}\right)$ is isomorphic to a quotient group of $\pi_{2}(X)$, and hence abelian; so are all higher relative homology groups. Since $H_{k}\left(X, M_{0}\right)=0$, the kernel of $h_{k}$ is the full group.

The action of $\pi_{1}$, by its definition, commutes with the boundary homomorphism $\partial: \pi_{k}\left(X, M_{0}\right) \rightarrow \pi_{k-1}\left(M_{0}\right)$. Hence

$$
\partial(\gamma(\eta)-\eta)=\gamma(\partial \eta)-\partial \eta=0
$$

as $M_{0}$ is a simple space. Thus, $\partial$ is the zero homomorphism.

Consider the commutative diagram

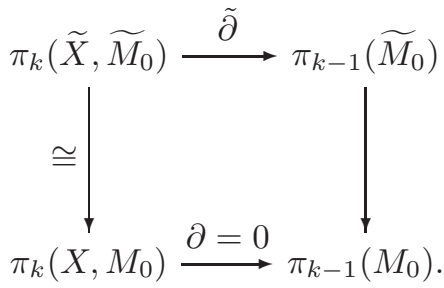

The vertical homomorphism on the left is an isomorphism for all $k \geq 2$. For $k \geq 3$, this is a general consequence of the five-lemma; for $k=2$ one needs to use that $\partial=0$. The vertical homomorphism on the right is an isomorphism for $k \geq 3$; for $k=2$ we have $\pi_{k-1}\left(\widetilde{M}_{0}\right)=0$. In either case we conclude that $\tilde{\partial}$ is also the zero homomorphism. 
Next we consider the commutative diagram coming from the 'homotopy-homology ladder' of the pair $\left(\widetilde{X}, \widetilde{M}_{0}\right)$ :

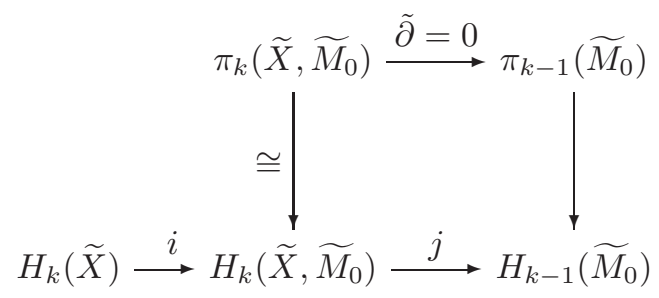

The vertical isomorphism on the left is the Hurewicz isomorphism. The commutative square implies the triviality of the homomorphism $j$. The homomorphism $i$ was shown to be trivial in the proof of Lemma 6.3. We conclude $H_{k}\left(\widetilde{X}, \widetilde{M}_{0}\right)=0$, hence $\pi_{k}\left(X, M_{0}\right)=\pi_{k}\left(\widetilde{X}, \widetilde{M}_{0}\right)=0$.

Lemma 8.2. The relative homotopy groups $\pi_{k}\left(X, M_{1}\right)$ are trivial.

Proof. Again we argue inductively; the inductive assumption for $k=0,1$ is satisfied. Assume that $\pi_{i}\left(X, M_{1}\right)$ vanishes for $i \leq k-1$. From the preceding lemma we know that $M_{0}$ is a deformation retract of $X$. It follows that $X$ is likewise a simple space. As in the foregoing proof we see that $\pi_{k}\left(X, M_{1}\right)$ is generated by elements of the form $\gamma(\zeta)-\zeta$ with $\zeta \in \pi_{k}\left(X, M_{1}\right)$ and $\gamma \in \pi_{1}\left(M_{1}\right)$. Again, $M_{1}$ being simple implies the triviality of the boundary homomorphism $\partial: \pi_{k}\left(X, M_{1}\right) \rightarrow \pi_{k-1}\left(M_{1}\right)$. Thus, the homomorphism $\pi_{k}(X) \rightarrow \pi_{k}\left(X, M_{1}\right)$ is surjective. It follows that $\left(X, M_{1}\right)$ is simple. With the information on the generators of $\pi_{k}\left(X, M_{1}\right)$, this shows that $\pi_{k}\left(X, M_{1}\right)$ is trivial.

Proof of Theorem 1.7. The two lemmata show that $\left\{M_{0}, X, M_{1}\right\}$ is an $h$-cobordism, and hence an $s$-cobordism under the assumption $\mathrm{Wh}\left(\pi_{1}(M)\right)=0$.

For an application of this theorem see Example 9.3

\section{Unit STABILISED COTANGENT BUNDLES}

We now return to contact manifolds of the kind described in Example 3.7. Given a closed Riemannian manifold $Q$ of dimension $q$, consider the unit sphere bundle $M:=S\left(T^{*} Q \oplus \mathbb{C}^{m}\right), m \geq 1$, of the $m$-fold stabilised cotangent bundle of $Q$. Then $\operatorname{dim} M=2 n-1$ with $n=q+m$. We always equip this manifold with the canonical contact structure $\xi$ given by the contact form

$$
\lambda_{Q}+\frac{1}{2} \sum_{j=1}^{m}\left(x_{j} \mathrm{~d} y_{j}-y_{j} \mathrm{~d} x_{j}\right) .
$$

Theorem 9.1. Let $M=S\left(T^{*} Q \oplus \mathbb{C}^{m}\right), m \geq 1$, with its standard contact structure $\xi$, where $Q$ is a closed q-dimensional manifold subject to the following conditions:

(i) $Q$ is aspherical, i.e. the universal cover $\widetilde{Q}$ is contractible.

(ii) The fundamental group $\pi_{1}(Q)$ is abelian and it has trivial Whitehead group $\mathrm{Wh}\left(\pi_{1}(Q)\right)$.

(iii) $n=q+m \geq 3$, that is, $\operatorname{dim} M \geq 5$.

Then every symplectically aspherical filling of $(M, \xi)$ is diffeomorphic to the total space of the disc bundle $W_{0}:=D\left(T^{*} Q \oplus \mathbb{C}^{m}\right)$. 
Proof. The assumption (ii) on $\pi_{1}(Q)$ being abelian implies that the inclusion $M \rightarrow$ $W$, for any symplectically aspherical filling $(W, \omega)$ of $(M, \xi)$, is $\pi_{1}$-isomorphic by Theorem 1.2 (b), and so all maps in the cobordism diagram are $\pi_{1}$-isomorphic by Lemma 5.1 and its proof. This allows us to pass to universal covers in that diagram.

Remark 9.2. If the assumption on $\pi_{1}(Q)$ being abelian is dropped, the conclusion of the theorem still holds true for all fillings $(W, \omega)$ for which the inclusion $M \rightarrow W$ is $\pi_{1}$-injective.

Since $\widetilde{Q}$ is contractible $(\widetilde{Q} \simeq *)$ by assumption (i), we also have $\widetilde{W}_{0} \simeq *$. Proposition 6.2 then tells us that $\widetilde{W} \simeq *$. From the homology sequence of the pair $\left(\widetilde{W}_{1}, \widetilde{W}_{0}\right)$, where $W_{1}$ is the diffeomorphic copy of $W$ in the notation of the preceding sections, we see that $H_{k}\left(\widetilde{W}_{1}, \widetilde{W}_{0}\right)=0$ in all degrees, and $H_{k}\left(\widetilde{X}, \widetilde{M}_{0}\right)=0$ by excision.

Thus, as in the proof of Theorem 7.2 we find that $M_{0}$ is a strong deformation retract of $X$. In particular, we have $H_{k}\left(X, M_{0}\right)=0$ for all $k$, and hence $H_{k}\left(X, M_{1}\right)=0$ for all $k$ by Poincaré duality and the universal coefficient theorem.

In order to show that the 'upper' inclusion $M_{1} \rightarrow X$ is likewise a homotopy equivalence, we need to establish $\pi_{k}\left(X, M_{1}\right)=0$ for all $k$; we already know this for $k=0,1$. To this end, analogous to the proof of Theorem 7.2 we have to show the vanishing of the relative homology groups $H_{k}\left(\widetilde{X}, \widetilde{M}_{1}\right)$.

From $H_{k}\left(\widetilde{X}, \widetilde{M}_{0}\right)=0$ for all $k$ we know that $H_{k}(\widetilde{X})$ is isomorphic to $H_{k}(\widetilde{M})$ for all $k$. Hence, if $H_{k}(\widetilde{M})=0$, then $H_{k}(\widetilde{X})=0$, and the inclusion $\widetilde{M}_{1} \rightarrow \widetilde{X}$ obviously induces an isomorphism on $H_{k}$; the same is true on $H_{0}$. In our situation, the only non-zero homology group of $\widetilde{M}$ in higher degree is $H_{q+2 m-1}(\widetilde{M}) \cong \mathbb{Z}$.

From the Gysin homology sequence of the sphere bundle $M \rightarrow Q$ we see that $H_{q+2 m-1}(M) \cong \mathbb{Z}$, generated by the fibre class. The same is true for the universal cover $\widetilde{M}$, which implies that the homomorphism

$$
\mathbb{Z} \cong H_{q+2 m-1}(\widetilde{M}) \longrightarrow H_{q+2 m-1}(M) \cong \mathbb{Z}
$$

is an isomorphism. The relevant part of the homology exact sequences of the pairs $\left(\widetilde{X}, \widetilde{M}_{0}\right)$ and $\left(X, M_{0}\right)$ becomes

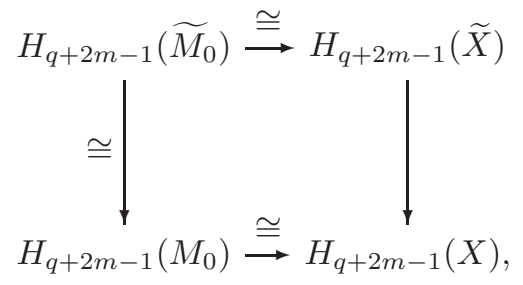

so the homomorphism

$$
\mathbb{Z} \cong H_{q+2 m-1}(\widetilde{X}) \longrightarrow H_{q+2 m-1}(X) \cong \mathbb{Z}
$$

is likewise an isomorphism. 
Finally, from the homology exact sequences of the pairs $\left(\widetilde{X}, \widetilde{M}_{1}\right)$ and $\left(X, M_{1}\right)$ we have

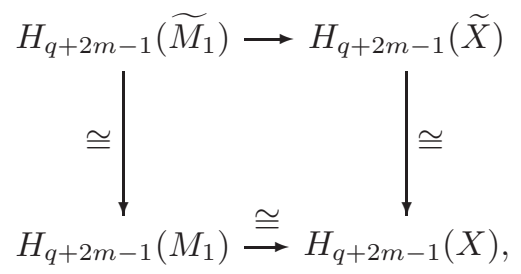

which gives us an isomorphism

$$
\mathbb{Z} \cong H_{q+2 m-1}\left(\widetilde{M}_{1}\right) \longrightarrow H_{q+2 m-1}(\widetilde{X}) \cong \mathbb{Z}
$$

Thus, the inclusion $\widetilde{M}_{1} \rightarrow \widetilde{X}$ is a homology isomorphism.

It follows that $\left\{M_{0}, X, M_{1}\right\}$ is an $h$-cobordism. Under the assumption that $\mathrm{Wh}\left(\pi_{1}(Q)\right)=0$ it is an $s$-cobordism, and hence trivial under the dimension assumption (iii).

Example 9.3. (1) Any closed Riemannian manifold $Q$ with abelian fundamental group and non-positive sectional curvature satisfies the assumptions of the theorem, since $Q$ is aspherical by the Hadamard-Cartan theorem, and $\mathrm{Wh}\left(\pi_{1}(Q)\right)$ is trivial by the work of Farrell and Jones [12.

(2) The conclusions of the theorem hold whenever $Q$ is a product of unitary groups and spheres. Indeed, in this case $\pi_{1}(M) \cong \pi_{1}(Q)$ is a free abelian group, which has trivial Whitehead group. The manifold $Q$ has a trivial stable tangent bundle, and $Q$ is a simple space, hence so is $M \cong Q \times S^{q+2 m-1}$. Then appeal to Theorem 1.7.

When information is given on the handle structure of the filling, as in the next theorem, we can use the results from Section 7 to remove the condition on $\pi_{1}(Q)$ being abelian.

Theorem 9.4. Let $M=S\left(T^{*} Q \oplus \mathbb{C}^{m}\right), m \geq 1$, with its standard contact structure $\xi$, where $Q$ is a closed $q$-dimensional manifold subject to the following conditions:

(i) $Q$ is aspherical.

(ii) $\mathrm{Wh}\left(\pi_{1}(Q)\right)$ is trivial.

(iii) $n=q+m \geq 3$, that is, $\operatorname{dim} M \geq 5$.

Then the following holds:

(a) All subcritical Stein fillings of $M$ are diffeomorphic to $W_{0}:=D\left(T^{*} Q \oplus \mathbb{C}^{m}\right)$.

(b) If $m \geq 2$, then all Stein fillings of $M$ are diffeomorphic to $W_{0}$.

Proof. In the notation of Lemma 7.1 we have $\ell_{0}=q \leq n-1$ and $\ell \leq n-1$ in case (a); $\ell_{0}=q \leq n-2$ and $\ell \leq n$ in case (b). In either case, the inclusion $M \rightarrow W$ (where $W$ is any filling of the described type) is $\pi_{1}$-isomorphic by Lemma 7.1.

Also, the assumption of Theorem 7.2 is satisfied, so the argument there shows that the 'lower' inclusion $M_{0} \rightarrow X$ in the cobordism $\left\{M_{0}, X, M_{1}\right\}$ is a homotopy equivalence.

The argument for the 'upper' inclusion $M_{1} \rightarrow X$ is then as in the preceding proof. 
Example 9.5. The conclusions of Theorem 9.4 hold for the following manifolds $Q$.

(1) Any closed surface. For orientable surfaces of genus at least 1 and nonorientable surfaces of genus at least 2, Theorem 9.4 applies directly thanks to the results cited in Example 9.3. For $Q=S^{2}$ even Theorem 9.1 holds true; this example is covered by Theorem 1.5.

For $Q=\mathbb{R} \mathrm{P}^{2}$, the Whitehead group of the fundamental group $\pi_{1}\left(\mathbb{R P}^{2}\right)=\mathbb{Z}_{2}$ vanishes. We claim that, again, Theorem 9.1 holds true in this case. Indeed, the only part of the argument that needs to be adapted is where we show that $H_{k}\left(\widetilde{X}, \widetilde{M}_{1}\right)$ vanishes for all $k$. As long as we only pass to finite covers $X^{\prime}, M_{1}^{\prime}$, this vanishing result holds by Poincaré duality. Thus, for the argument in the proof of Theorem 9.1 to go through it suffices to find a finite cover $M_{1}^{\prime}$ of $M_{1}$ such that the projection map $\widetilde{M}_{1} \rightarrow M_{1}^{\prime}$ induces an isomorphism on $H_{k}$ whenever $H_{k}\left(\widetilde{M}_{1}\right)$ is nontrivial. In the present example, we can take $M_{1}^{\prime}=\widetilde{M}_{1}=S^{2} \times S^{2 m+1}$. Of course, in this case of a finite fundamental group we can alternatively appeal directly to Theorem 5.3 .

(2) Any closed, irreducible aspherical 3-manifold. This follows from the result of Roushon 37 that the fundamental group of such a manifold has a trivial Whitehead group.

(3) Any closed, irreducible 3-manifold $Q$ covered by $S^{3}$ with $\mathrm{Wh}\left(\pi_{1}(Q)\right)=0$. Here the argument is as in (1). Examples of the allowed fundamental groups are $\mathbb{Z}_{2}, \mathbb{Z}_{3}, \mathbb{Z}_{4}, \mathbb{Z}_{6}$.

\section{Symplectomorphism type}

We now assume that $(M, \xi)$ is a closed, connected contact manifold of dimension $2 n-1, n \geq 3$, that admits a 2 -subcritical Stein filling $\left(W_{0}, \omega_{0}\right)$, that is, where the Stein handles are all of index at most $n-2$. In particular, we have $\ell_{0} \leq n-2$. Under this assumption, we want to formulate topological conditions on $M$ that allow us to classify all subcritical fillings up to symplectomorphism.

For the notion of Stein deformation equivalence see [9, p. 311]. Deformation equivalent Stein fillings are symplectomorphic in the sense of [9. p. 318]. The concept of flexible Stein fillings is defined in [9, Definition 11.29]. Suffice it to say here that all subcritical Stein fillings are flexible.

Theorem 10.1. Let $(M, \xi)$ be a $(2 n-1)$-dimensional closed, connected contact manifold, $n \geq 3$, admitting a 2-subcritical Stein filling $\left(W_{0}, \omega_{0}\right)$. Further, we make the following topological assumptions:

(i) $\operatorname{Wh}\left(\pi_{1}(M)\right)=0$.

(ii) $M$ (or some finite cover of $M$ ) is a simple space, or $M$ (or some finite cover) has the property that the homomorphism $H_{k}(\widetilde{M}) \rightarrow H_{k}(M)$ is an isomorphism whenever $H_{k}(\widetilde{M}) \neq 0$.

Then all flexible Stein fillings of $(M, \xi)$ are Stein deformation equivalent.

Example 10.2. The assumptions of the theorem are satisfied by $\left(S^{2 n-1}, \xi_{\mathrm{st}}\right)$ and by any sphere bundle $S\left(T^{*} Q \oplus \mathbb{C}^{m}\right)$ with its standard contact structure, provided $m \geq 2, \mathrm{Wh}\left(\pi_{1}(Q)\right)=0$, and $Q$ is a Lie group or satisfies the homological assumption (ii) on coverings.

Proof of Theorem 10.1. Given a flexible Stein filling $(W, \omega)$ of $(M, \xi)$, we consider the cobordism $\left\{M_{0}, X, M_{1}\right\}$ as in Section 5 . As we saw in the proof of Theorem 7.2. 
the lower inclusion $M_{0} \rightarrow X$ is a homotopy equivalence. The topological condition (ii) guarantees that the upper inclusion is likewise a homotopy equivalence, see Section 8 for the case that $M$ is a simple space, and the argument in Example 9.5 (2) for the case when the homological information on $\widetilde{M}$ is given. Together with condition (i) this implies that $\left\{M_{0}, X, M_{1}\right\}$ is an $s$-cobordism, so that $W$ and $W_{0}$ are diffeomorphic.

We now want to construct a Stein structure on the cobordism $\left\{M_{0}, X, M_{1}\right\}$. To this end, consider a Stein structure on $\mathbb{C}$ with two 0-handles and one 1-handle; the gradient flow of the plurisubharmonic potential is shown in Figure 3 . The assumption on $W_{0}$ being 2 -subcritical translates into saying that the Stein manifold $\left(V, J_{V}\right)$ — in the notation of Section 2 - has a plurisubharmonic potential $\psi_{V}$ with finitely many critical points up to index $n-2$ only. On the product $V \times \mathbb{C}$ we then still have a subcritical potential $\psi$. Each critical point of $\psi_{V}$ gives rise to three critical points of $\psi$; the ones sitting over the index 1 point in $\mathbb{C}$ have their index shifted up by 1 .

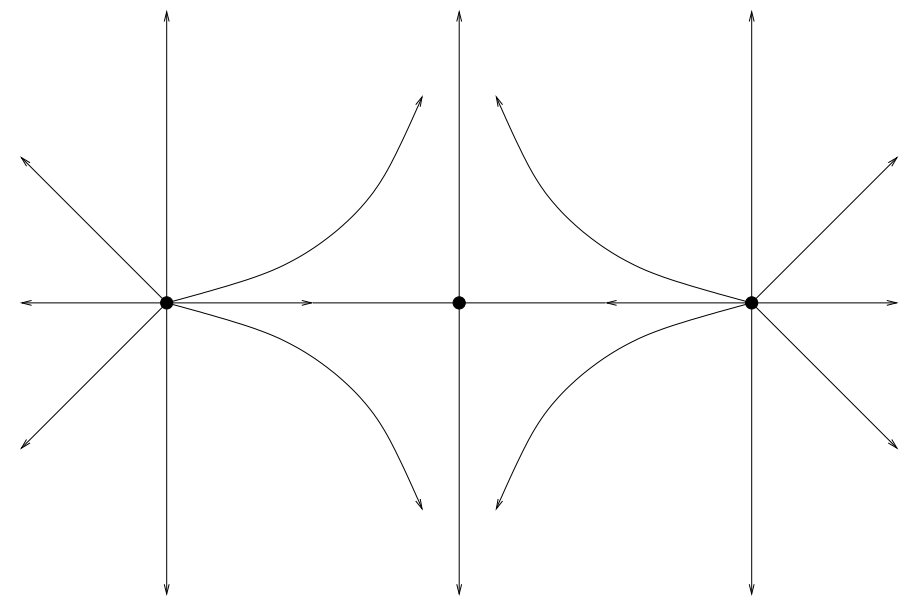

Figure 3. A Stein structure on $\mathbb{C}$.

Now we build the cobordism $X$ as before, where the copy of $W_{0}$ that is replaced by $W$ and the copy of $W_{0}$ that is removed are each placed, in the $\mathbb{C}$-direction, in a neighbourhood of one of the critical points of index 0. This induces a Stein structure on the cobordism. This structure is flexible since, apart from the flexible handles coming from $W$, it only contains subcritical handles. So the theorem follows from the Stein $h$-cobordism theorem [9, Corollary 15.12].

Acknowledgements. We thank Paul Biran and Dietmar Salamon for useful conversations. The commutative diagrams have been produced with Paul Taylor's TEX macros.

\section{REFERENCES}

[1] P. Albers and U. Frauenfelder, Rabinowitz Floer homology: a survey, in Global Differential Geometry, Springer Proc. Math. 17, Springer-Verlag, Berlin (2012), 437-461.

[2] M. Audin, F. Lalonde And L. Polterovich, Symplectic rigidity: Lagrangian submanifolds, in Holomorphic Curves in Symplectic Geometry, Progr. Math. 117, Birkhäuser Verlag, Basel (1994), 271-321. 
[3] R. Bott and L. W. Tu, Differential Forms in Algebraic Topology, Grad. Texts in Math. 82, Springer-Verlag, New York (1982).

[4] G. E. Bredon, Topology and Geometry, Grad. Texts in Math. 139, Springer-Verlag, New York (1993).

[5] C. Caubel, A. Némethi and P. Popescu-Pampu, Milnor open books and Milnor fillable contact 3-manifolds, Topology 45 (2006), 673-689.

[6] R. Chinang, F. Ding and O. van Koert, Open books for Boothby-Wang bundles, fibered Dehn twists and the mean Euler characteristic, J. Symplectic Geom. 12 (2014), 379-426.

[7] R. Chiang, F. Ding and O. van Koert, Non-fillable invariant contact structures on principal circle bundles and left-handed Dehn twists, Internat. J. Math. 27 (2016), 1650024, 55 pp.

[8] K. Cieliebak, Subcritical Stein manifolds are split, arXiv:math/020435.

[9] K. Cieliebak and Ya. Eliashberg, From Stein to Weinstein and Back - Symplectic geometry of affine complex manifolds, Amer. Math. Soc. Colloq. Publ. 59, American Mathematical Society, Providence, RI (2012).

[10] K. Cieliebak, U. Frauenfelder and A. Oancea, Rabinowitz Floer homology and symplectic homology, Ann. Sci. Éc. Norm. Supér. (4) 43 (2010), 957-1015.

[11] K. Cieliebak And K. Mohnke, Symplectic hypersurfaces and transversality in GromovWitten theory, J. Symplectic Geom. 5 (2007), 281-356.

[12] F. T. Farrell and L. E. Jones, Rigidity and other topological aspects of compact nonpositively curved manifolds, Bull. Amer. Math. Soc. (N.S.) 22 (1990), 59-64.

[13] H. Geiges, Applications of contact surgery, Topology 36 (1997), 1193-1220.

[14] H. Geiges, An Introduction to Contact Topology, Cambridge Stud. Adv. Math. 109, Cambridge University Press (2008).

[15] H. Geiges, Contact structures and geometric topology, in Global Differential Geometry, Springer Proc. Math. 17, Springer-Verlag, Berlin (2012), 463-489.

[16] H. Geiges and K. Zehmisch, Symplectic cobordisms and the strong Weinstein conjecture, Math. Proc. Cambridge Philos. Soc. 153 (2012), 261-279.

[17] H. Geiges and K. Zehmisch, Reeb dynamics detects odd balls, Ann. Sc. Norm. Super. Pisa Cl. Sci. (5) 15 (2016), 663-681.

[18] H. Geiges and K. Zenmisch, The Weinstein conjecture for connected sums, Int. Math. Res. Not. IMRN 2016 (2016), 325-342.

[19] P. Ghiggini, K. Niederkrüger and C. Wend, Subcritical contact surgeries and the topology of symplectic fillings, J. Éc. polytech. Math. 3 (2016), 163-208.

[20] M. Gromov, Pseudoholomorphic curves in symplectic manifolds, Invent. Math. 82 (1985), $307-347$.

[21] A. Hatcher, Algebraic Topology, Cambridge University Press (2002).

[22] M. W. Hinsch, Differential Topology, Grad. Texts in Math. 33, Springer-Verlag, New York (1976).

[23] O. VAN KoeRT, Lecture notes on stabilization of contact open books, arXiv: 1012.4359.

[24] O. VAN KOERT AND K. NiedERKRÜGER, Open book decompositions for contact structures on Brieskorn manifolds, Proc. Amer. Math. Soc. 133 (2005), 3679-3686.

[25] P. LiscA, On lens spaces and their symplectic fillings, Math. Res. Lett. 11 (2004), 13-22.

[26] J. McCleary, A User's Guide to Spectral Sequences, Cambridge Stud. Adv. Math. 58, Cambridge University Press (2001).

[27] D. McDuff, The structure of rational and ruled symplectic 4-manifolds, J. Amer. Math. Soc. 3 (1990), 679-712.

[28] D. McDuff, Symplectic manifolds with contact type boundaries, Invent. Math. 103 (1991), $651-671$.

[29] D. McDuff and D. Salamon, Introduction to Symplectic Topology, 2nd edition, Oxford Math. Monogr., Oxford University Press (1998).

[30] D. McDuff and D. Salamon, J-holomorphic Curves and Symplectic Topology, Amer. Math. Soc. Colloq. Publ. 52, American Mathematical Society, Providence, RI (2004).

[31] M. McLean, Lefschetz fibrations and symplectic homology, Geom. Topol. 13 (2009), 18771944.

[32] J. Milnor, Lectures on the h-Cobordism Theorem, Princeton University Press, Princeton, NJ (1965).

[33] J. Milnor, Whitehead torsion, Bull. Amer. Math. Soc. 72 (1966), 358-426. 
[34] J. Milnor, Singular Points of Complex Hypersurfaces, Ann. of Math. Stud. 61, Princeton University Press, Princeton, NJ (1968).

[35] A. Oancea and C. Viterbo, On the topology of fillings of contact manifolds and applications, Comment. Math. Helv. 87 (2012), 41-69.

[36] B. ÖzBaĞCi And A. I. Stipsicz, Surgery on Contact 3-Manifolds and Stein Surfaces, Bolyai Soc. Math. Stud. 13, Springer-Verlag, Berlin; János Bolyai Mathematical Society, Budapest (2004).

[37] S. K. Roushon, Vanishing structure set of 3-manifolds, Topology Appl. 158 (2011), 810-812.

[38] P. SeIdel, Lagrangian two-spheres can be symplectically knotted, J. Differential Geom. 52 (1999), 145-171.

[39] P. Seidel, Lectures on four-dimensional Dehn twists, in Symplectic 4-Manifolds and Algebraic Surfaces, Lecture Notes in Math. 1938, Springer-Verlag, Berlin (2008), 231-267.

[40] P. Seidel, Exotic iterated Dehn twists, Algebr. Geom. Topol. 14 (2014), 3305-3324.

[41] P. Seidel and I. Smith, The symplectic topology of Ramanujam's surface, Comment. Math. Helv. 80 (2005), 859-881.

[42] I. UlJAREviĆ, Floer homology of automorphisms of Liouville domains, arXiv: 1404.2128.

[43] I. UlJarević, A symplectic homology theory for automorphisms of Liouville domains, Ph.D. thesis, ETH Zürich (2016).

Mathematisches Institut, WWU Münster, Einsteinstrasse 62, 48149 Münster, GerMANY

E-mail address: k_bart05@uni-muenster.de

Mathematisches Institut, Universität Zu Köln, Weyertal 86-90, 50931 Köln, GerMANY

E-mail address: geiges@math.uni-koeln.de

Mathematisches Institut, WWU Münster, Einsteinstrasse 62, 48149 Münster, GerMANY

E-mail address: kai.zehmisch@uni-muenster.de 